\title{
The DNAPKcs long-range C-NHEJ complex is required for blunt DNA end joining when XLF is weakened
}

Metztli Cisneros-Aguirre ${ }^{1,2}$, Felicia Wednesday Lopezcolorado ${ }^{1}$, Linda Jillianne Tsai ${ }^{1,2}$, Ragini Bhargava ${ }^{1,2,3}$, and Jeremy M. Stark ${ }^{1,2,4}$

${ }^{1}$ Department of Cancer Genetics and Epigenetics, ${ }^{2}$ Irell and Manella Graduate School of Biological Sciences Beckman Research Institute of the City of Hope, 1500 E Duarte Rd., Duarte, CA 91010 USA, ${ }^{3}$ Current address Department of Pharmacology and Chemical Biology, UPMC Hillman Cancer Center, University of Pittsburgh, Pittsburgh, PA, USA

${ }^{4}$ Correspondence should be addressed to J.M.S:

Phone: 626-218-6346

Fax: 626-301-8892

jstark@coh.org

Key Words: non-homologous end joining; XLF; DNAPKes; double strand break repair. 


\begin{abstract}
Canonical non-homologous end joining (C-NHEJ) factors can assemble into a long-range (LR) complex with DNA ends relatively far apart that contains DNAPKcs, XLF, XRCC4, LIG4, and the KU heterodimer and a short-range (SR) complex lacking DNAPKcs that has the ends positioned for ligation. Since the SR complex can form de novo, the role of the LR complex (i.e., DNAPKcs) for chromosomal EJ is unclear. We have examined EJ of chromosomal blunt DNA double-strand breaks (DSBs), and found that DNAPKcs is significantly less important than XLF and XRCC4 for such EJ. However, weakening XLF via disrupting interaction interfaces (e.g., disrupting the XLF homodimer interface) causes a marked requirement for DNAPKcs, its kinase activity, and its ABCDE-cluster autophosphorylation sites for blunt DSB EJ. In contrast, other aspects of genome maintenance are sensitive to DNAPKcs kinase inhibition in a manner that is not further enhanced by XLF loss (i.e., suppression of homology-directed repair and structural variants, and IR-resistance). We suggest that DNAPKcs is required to position a weakened XLF in an LR complex that can transition into a functional SR complex for blunt DSB EJ, but also has distinct functions for other aspects of genome maintenance.
\end{abstract}




\section{INTRODUCTION}

Repair of DNA double-strand breaks (DSBs) is critical for genome stability, and is a central aspect of genome editing ${ }^{1-3}$. DNA DSB repair is also a mechanism of cancer cell therapeutic resistance to clastogenic agents, such as ionizing radiation (IR) ${ }^{4}$. Canonical non-homologous end joining (C-NHEJ), is a major DSB repair pathway that functions throughout the cell cycle, and involves the factors KU70/80 (KU), XRCC4, DNA Ligase 4 (LIG4), XLF, and DNAPKcs ${ }^{5,6}$. Accordingly, characterizing the mechanism of C-NHEJ provides insight into genome maintenance, tumor resistance to clastogenic therapeutics, and gene editing.

A central aspect of C-NHEJ is synapsis of the two DNA ends to enable efficient ligation. Single molecule studies in Xenopus extracts identified both a long-range (LR) synapsis interaction between labeled DNA molecules without fluorescence resonance energy transfer (FRET), and a short-range (SR) synapsis interaction with detection of FRET between the labels7. Recent cryoelectron microscopy (cryo-EM) structures have identified complexes that can mediate such LR and SR interactions ${ }^{8,9}$. For one, DNAPKcs and KU bound to each DNA end are able to support an LR interaction ${ }^{9}$. However, an additional complex has been identified that also includes XRCC4, XLF, and LIG4, which we will refer to as the LR complex ${ }^{8,9}$. For this LR complex, each DNA end is bound to one molecule of DNAPKcs, KU, XRCC4, and LIG4 ${ }^{8,9}$. In addition, a single XLF homodimer appears to bridge the two DNA ends through interactions of each XLF monomer with XRCC4 and $\mathrm{KU}^{8,9}$. As mentioned above, the DNA ends in this complex are not close enough to facilitate ligation. However, cryo-EM studies have also identified an SR complex with DNA ends positioned to enable ligation ${ }^{8}$. This SR complex has 
commonalities with the LR complex, in that it also contains a single XLF homodimer bridging two DNA ends that are each bound to a molecule of XRCC4, KU, and LIG4 ${ }^{8}$. However, this SR complex does not contain DNAPKcs ${ }^{8}$.

Whether the LR complex is a prerequisite to form a functional SR complex in cells remains controversial. From studies in Xenopus extracts, DNAPKcs is important not only to form the LR interaction, but also for the transition to the SR interaction, in a manner dependent on DNAPKcs kinase activity ${ }^{7}$. However, using purified proteins the SR complex appears to form without DNAPKcs. Namely, as mentioned above, the cryo-EM structure of the SR complex was generated without DNAPKcs ${ }^{8}$. Similarly, also with purified proteins, close end synapsis interactions have been shown to form with KU, XLF, XRCC4 and LIG4 without requiring DNAPKcs ${ }^{10,11}$. These studies raise the notion that under some circumstances the SR complex can form de novo, i.e., without prior formation of the LR complex that contains DNAPKes.

Furthermore, the requirement for the DNAPKcs LR complex for chromosomal DSB EJ has remained unclear, particularly outside the context of V(D)J recombination. $\mathrm{V}(\mathrm{D}) \mathrm{J}$ recombination is a programmed rearrangement that involves joining of both hairpin coding ends and signal ends ${ }^{12,13}$. For V(D)J recombination, DNAPKcs is important to recruit the Artemis nuclease for coding EJ, promotes both coding and signal EJ of plasmid substrates, but is not essential for chromosomal signal EJ in mouse lymphocytes unless paired with loss of other factors (i.e., XLF or the ATM kinase) ${ }^{12,14-17}$. Separate from this programmed C-NHEJ event, the role of DNAPKcs on chromosomal EJ is poorly understood. Indeed, recent studies have suggested that DNAPKcs largely functions during genome maintenance to regulate cellular senescence and chromatin 
decondensation, as well as playing a role in rRNA processing ${ }^{18-20}$. This gap in knowledge on the role of DNAPKes for chromosomal EJ is partly due to limitations of DSB repair assays that measure chromosomal rearrangements and/or insertion/deletion mutations (indels) that can also be mediated by the Alternative EJ pathway ${ }^{13,21-24}$. However recent studies using the RNA guided nuclease Cas9, which largely induces blunt DSBs ${ }^{25-27}$, have found that EJ of two Cas9 blunt DSBs without indels (No Indel EJ) is markedly dependent on C-NHEJ (i.e., XRCC4, LIG4, XLF, and KU) ${ }^{28-30}$. Thus, we have sought to define the role of DNAPKcs for EJ of blunt chromosomal DSBs, as well as other DSB repair outcomes, both on its own and in the context of mutants of XLF. 


\section{RESULTS}

DNAPKes is less important for No Indel EJ vs. XLF and XRCC4, but becomes essential in combination with an XLF mutant (K160D).

We have sought to understand the role of DNAPKcs on EJ outcomes, with a focus on EJ of blunt chromosomal DSBs. This type of EJ does not involve an annealing intermediate that could facilitate Alternative EJ, and therefore likely requires stable DSB end bridging via the C-NHEJ complex to facilitate efficient ligation. To begin with, we used the EJ7-GFP reporter (Fig 1a) that measures EJ of blunt DNA DSBs without indels (No Indel EJ). For these experiments, EJ7-GFP was chromosomally integrated into human HEK293 cells, mouse embryonic stem cells (mESCs), and human U2OS cells. This reporter contains a green fluorescent protein (GFP) sequence interrupted by a 46 bp spacer at a glycine codon critical for GFP fluorescence (Fig 1a). We induced two blunt DSBs at the edges of the 46 bp spacer using Cas9 and two single guide RNAs (sgRNAs), which are introduced by transfection of expression plasmids. If the distal blunt DSB ends are repaired via No Indel EJ, this repair event leads to GFP expressing cells that are detected via flow cytometry. Using this assay, No Indel EJ was shown to be markedly promoted by the CNHEJ factors XRCC4, XLF, and $\mathrm{KU} 70^{30}$, and we sought here to examine the influence of DNAPKcs (PRKDC gene) on such EJ.

Beginning with HEK293 cells with the EJ7-GFP reporter, we examined several knockout $(\mathrm{KO})$ cell lines and found that there was a significant decrease in No Indel EJ in $P R K D C-K O, X L F-K O$, and $X R C C 4-K O$ cells compared to the parental HEK293 cells (Fig 1b). However, for $P R K D C-K O$ cells, the No Indel EJ frequency was significantly higher compared to $X L F-K O$ cells, which itself was significantly higher compared to $X R C C 4-K O$ 
cells (Fig 1b). We also found similar results with U2OS cells (Supplemental Fig S1a). Thus, DNAPKcs appears relatively less important for No Indel EJ compared to XLF and XRCC4 in two human cell lines. We then posited that the residual No Indel EJ in both the $P R K D C-K O$ and $X L F-K O$ cell lines is due to partial functional redundancies between DNAPKcs and the XLF homodimer (Fig 1c $)^{31}$ to meditate DNA end bridging ${ }^{8,9,32}$. To begin to test this hypothesis, we examined No Indel EJ in $X L F-K O / P R K D C-K O$ double mutant HEK293 cells, and found that No Indel EJ was ablated compared to the $X L F-K O$ cells (Fig 1d).

We next posited that weakening XLF binding interfaces that could mediate DSB end bridging might reveal a critical role for DNAPKcs in No Indel EJ. In particular, given that a single XLF homodimer has been shown in recent cryo-EM structures to bridge DNA ends via interactions with other proteins in the C-NHEJ complex ${ }^{8,9}$, we tested whether a mutant that weakens the dimer interface may reveal a greater role for DNAPKcs. Specifically, we mutated the K160 residue, which is within the coiled-coil dimerization interface and is predicted to form a salt bridge with the D161 residue on the other monomer (Fig 1c) ${ }^{30,31,33}$. Thus, a XLF-K160D mutant would be predicted to disrupt this salt bridge, and indeed has been shown to increase the coiled-coil interface distance within the XLF homodimer, based on molecular dynamics modeling ${ }^{30,31,33}$. Thus, we posited that the XLFK160D mutant would cause a greater dependency on DNAPKcs for No Indel EJ.

To test this hypothesis, we first compared the ability for transient expression of the control XLF-WT to promote No Indel EJ in the presence or absence of DNAPKcs (i.e., in $X L F-K O$ vs. $P R K C D-K O / X L F-K O)$, and found that loss of DNAPKcs caused a 5.5 -fold reduction in No Indel EJ (Fig 1d). We then compared XLF-WT vs. XLF-K160D in the 
presence of DNAPKcs (i.e., $X L F-K O$ ), and found that XLF-K160D caused a modest decrease in No Indel EJ vs. XLF-WT (1.6-fold). Thus, the XLF-K160D mutation has only a modest effect in the presence of DNAPKcs. However, when we examined the double mutant (XLF-K160D in XLF-KO/PRKDC-KO cells), we found a marked loss of No Indel EJ (Fig 1d, 172-fold decrease vs. XLF-KO cells complemented with XLF-WT). Notably, we also found that transient expression of DNAPKcs in the double-mutant (XLF-K160D in $X L F-K O / P R K D C-K O$ ) was able to substantially complement No Indel EJ (Fig 1d). Although, the levels of such EJ did not recover to the levels of the single mutant (XLF$\mathrm{K} 160 \mathrm{D}$ in $X L F-K O$ ) likely because the expression vector was unable to restore endogenous levels of DNAPKcs (Fig 1d). We also tested the effect of loss of DNAPKcs on No Indel EJ in mESCs with XLF-WT vs. XLF-K160D, and found similar results (Fig 1e). These findings indicate that the XLF-K160D mutant causes a marked requirement for DNAPKcs for No Indel EJ.

\section{Inhibiting DNAPKcs kinase activity suppresses No Indel EJ to a greater degree when combined with XLF-K160D.}

We then performed similar experiments with inhibition of DNAPKcs kinase

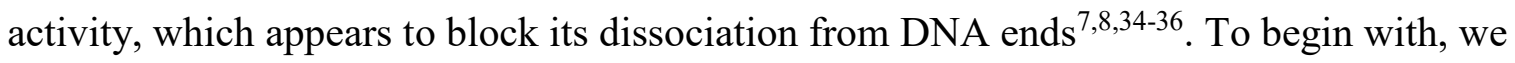
interrogated the effect of inhibiting DNAPKcs kinase activity with the small molecule inhibitor M3814 (i.e., Nedisertib) ${ }^{37}$, and found a concentration dependent decrease in No Indel EJ events in HEK293 (Fig 2a and Supplemental Fig 1b). Notably, we did not see a concentration dependent decrease in phosphorylation of DNAPKcs at S2056 via immunoblotting, suggesting the EJ7-GFP reporter for No Indel EJ is more sensitive to the concentration of M3814 (Fig 2b). We then examined the effect of M3814 on No Indel EJ 
in $X L F-K O$ cells expressing XLF-WT and found similar results to the parental HEK293 cells (Fig 2a). However, combining XLF-K160D and M3814 treatment caused a synergistic loss of No Indel EJ (Fig 2a). For example, in comparison to control cells (XLFWT in $X L F-K O), 500 \mathrm{nM} \mathrm{M} 3814$ treatment caused a 3.8-fold decrease, XLF-K160D without M3814 caused a 1.7-fold decrease, but the combination of XLF-K160D with 500 nM M3814 caused a 52-fold decrease (Fig 2b). We also tested the combination of XLFK160D and M3814 on No Indel EJ in $X L F-K O$ U2OS cells and again found synergistic effects (Fig 2c, Supplemental Fig S1b, c). These findings indicate that XLF-K160D causes an enhanced requirement for DNAPKcs kinase activity for No Indel EJ.

\section{Inhibiting DNAPKes kinase activity affects EJ junction patterns to a greater degree when combined with XLF-K160D.}

We next interrogated our above hypothesis using a distinct approach that enables measurement of diverse EJ outcomes: the GAPDH-CD4 rearrangement assay that uses endogenous genes in human chromosome $12^{28}$. This assay uses two sgRNAs to target Cas 9 DSBs after the promoter of both the GAPDH and CD4 genes, such that if there is a deletion rearrangement between the two DSBs (i.e., a GAPDH-CD4 rearrangement), CD4 is expressed from the GAPDH promoter (Fig 3a). These CD4+ cells are detected by immunostaining, and following isolation by fluorescence activated cell sorting (FACS), the GAPDH-CD4 rearrangement junctions are amplified and analyzed by deep sequencing.

To begin with, we examined the overall GAPDH-CD4 rearrangement frequency (i.e., percentage of $\mathrm{CD4}+$ ) in various HEK293 cell conditions. To examine DNAPKcs kinase inhibitor treatment, we used the concentration of $500 \mathrm{nM} \mathrm{M} 3814$, which had intermediate effects on the EJ7-GFP assay, compared to $250 \mathrm{nM}$ and $1000 \mathrm{nM}$ (Fig 2a). 
We found that such M3814 treatment caused a modest but significant decrease in CD4+ cells (Fig 3b). The PRKDC-KO and XLF-KO cells also showed a decrease in $C D 4+$ cells compared to parental cells, although the frequency for $P R K D C-K O$ was higher than $X L F$ $K O$ cells, indicating that XLF promotes this rearrangement more so than DNAPKcs. Next, we examined combinations of M3814 treatment with $X L F-K O$ cells with and without expression of XLF-WT and XLF-K160D. We found that M3814 had no effect on CD4+ frequencies for either $X L F-K O$ cells or $X L F-K O$ cells expressing XLF-WT (Fig 3c). However, for $X L F-K O$ cells expressing XLF-K160D, M3814 treatment caused a significant decrease in the $C D 4+$ cells, indicating DNAPKcs kinase activity is important for the GAPDH-CD4 rearrangement with XLF-K160D.

We then examined the frequency of distinct rearrangement junction types for each of the above conditions using amplicon deep sequencing of $C D 4+$ cells. Specifically, we classified the junctions as No Indel EJ (precise joining of the distal blunt Cas9 DSBs), deletions (loss of nucleotides), insertions (gain of nucleotides), or complex indel (combined loss/gain of nucleotides). We determined the frequency of each of these junction types based on read counts from $C D 4+$ sorted cells, for three independent transfections and sorted samples per condition. Accordingly, the statistical analysis is based on the frequency for each junction type from the triplicate samples (i.e., $n=3$ ).

From this analysis, we found that loss of DNAPKcs, as well as M3814 treatment caused a significant decrease in No Indel EJ, but the fold-effects were relatively modest (Fig 3d). In contrast, $X L F-K O$ cells showed a marked drop in No Indel EJ that was significantly lower than the $P R K D C-K O$ cells (Fig 3d). These findings are similar to the results with the EJ7-GFP reporter (Fig 1). Interestingly, the influence of DNAPKcs and 
XLF on insertions was similar to the effect on No Indel EJ (Fig 3d). However, we observed the converse effect on deletion mutations. Namely, PRKDC-KO cells, M3814 treatment of parental cells, and $X L F-K O$ cells each showed a significant increase in the frequency of deletions, although the effect of XLF loss was greater than that of DNAPKcs loss (Fig 3d). Finally, complex indel junctions were relatively infrequent for all of the conditions (Fig 3d).

We then examined junction types in $X L F-K O$ cells expressing XLF-WT or XLFK160D, each with and without M3814 treatment, using $500 \mathrm{nM}$ as above (Fig 3e). For the No Indel EJ and insertion junction types, cells with XLF-K160D showed a modest decrease (1.3-fold and 1.6-fold, respectively) compared to XLF-WT, which was similar to the effects of M3814 treatment with XLF-WT (both 1.5-fold, Fig 3e). Conversely, deletion junctions were increased (1.7-fold for XLF-K160D, 1.8-fold for M3814, Fig 3e). However, cells with XLF-K160D combined with M3814 treatment showed a synergistic decrease in No Indel EJ (8-fold), a similar synergistic decrease in insertions (16-fold), and a converse increase in deletions (3.2-fold, Fig 3e). In contrast, complex indel junctions were infrequent for all conditions (Fig 3e). These findings indicate that combining XLF-K160D with DNAPKcs kinase inhibition causes a synergistic decrease in both No Indel EJ and insertions, along with a converse increase in deletions.

\section{EJ with insertions that are likely caused by staggered Cas9 DSBs show similar genetic requirements as No Indel EJ.}

Given that insertions and No Indel EJ showed similar genetic requirements in the above junction analysis, we further evaluated the nature of such insertions. In particular, 
we considered whether the insertions may result from staggered Cas9 DSBs that cause 5' overhangs, which are relatively less common than Cas9 blunt DSBs, but nonetheless are readily detectable ${ }^{25}$. Accordingly, the insertions would be caused by filling-in the 5, overhangs, and subsequent EJ of the blunt DNA ends. For the GAPDH-CD4 rearrangement, staggered Cas9 DSBs at the GAPDH site would be expected to cause a 1 nt C-insertion and a 2 nt CG-insertion for 5' overhangs of 1 and 2 nt, respectively (Supplemental Fig S2a). To determine the frequency of these events, we analyzed the insertion junctions from the above experiment with HEK293 cells. We found that +1 and +2 insertions were predominant, and that the inserted nucleotides were almost entirely consistent with such staggered Cas9 DSBs (Supplemental Fig S2b, c). Furthermore, for the relatively rare $\geq 3 \mathrm{nt}$ insertions, approximately half of these junctions are also consistent with staggered Cas9 DSBs (Supplemental Fig S2c).

To examine such insertions with another approach, we adapted the EJ7-GFP reporter to examine $1 \mathrm{nt}$ insertions (+1 EJ, EJ7+1-GFP, Fig 4a). The reporter construct itself is the same as EJ7-GFP, as is the position of the $3^{\prime}$ DSB site, but the 5' DSB site is targeted $1 \mathrm{nt}$ upstream. Accordingly, GFP would be restored if Cas9 at the 5' site causes a staggered DSB with a $1 \mathrm{nt} 5$ ' overhang that is filled-in and then repaired by EJ with the distal 3' blunt DSB end (Fig 4a, Supplemental Fig S2d).

Using the EJ7+1-GFP reporter, we examined the influence of DNAPKcs and XLF on +1 -EJ events in HEK293 cells. With PRKDC-KO and XLF-KO cells, we found a significant decrease in such EJ vs. parental HEK293 cells, and that PRKDC-KO cells showed a significantly higher frequency vs. $X L F-K O$ cells (Fig $4 b)$. We next interrogated the influence of DNAPKes in combination with XLF-K160D, using both $X L F$ - 
KO/PRKDC-KO cells (Fig 4c) and M3814 treatment (Fig 4d). From this analysis, we found similar results as with No Indel EJ (Fig 1,2), in that combined loss of DNAPKes and XLFK160D caused a marked decrease of +1-EJ (Fig 4c), and M3814 treatment in XLF-K160D cells had a substantially greater effect on +1-EJ vs. XLF-WT expressing cells (Fig 4d). Since $+1-E J$ events are likely caused by blunt EJ after filling-in the 5' overhang of the staggered Cas9 5' DSB, and since No Indel EJ is also the product of blunt DSB EJ, we suggest that the XLF-K160D mutant causes a marked requirement for DNAPKcs and its kinase activity for blunt DSB EJ.

\section{DNAPKes becomes essential for No Indel EJ in combination with other XLF mutants with disrupted binding interfaces, and depends on the DNAPKcs-ABCDE}

\section{phosphorylation sites cluster.}

Recent cryo-EM structures of LR and SR C-NHEJ complexes, and other stoichiometric analyses, indicate that a single XLF homodimer can bridge two C-NHEJ complexes bound to DNA ends ${ }^{8,9,38}$. Specifically, the XLF homodimer appears to form this bridge via interactions of each XLF monomer with one molecule each of XRCC4 and $\mathrm{KU}^{8,9,38}$. In the above analysis, we used XLF-K160D that disrupts the homodimer interface $^{30}$, which we suggest likely weakens such bridging. We next posited that disrupting the interaction interfaces of XLF with XRCC4 and KU would also weaken XLFmediated bridging of two C-NHEJ complexes, and hence also reveal a greater requirement for DNAPKcs for No Indel EJ. To test this hypothesis, we examined four other XLF mutants: two that disrupt the leucine-lock interaction with XRCC4 (XLF-L115A, XLFL115D) $)^{30,31,38-42}$, and two that disrupt the KU Binding Motif ( $\triangle \mathrm{KBM}$ that deletes residues 
287-299, and XLF-4KA with four conserved lysine residues in the KBM mutated to alanine, which disrupts the interaction with $\mathrm{KU})^{30,43}$ (Fig 5a).

First, we examined the capacity for these various XLF mutants to promote No Indel EJ with and without M3814 treatment (500 nM, as described above), using XLF-KO HEK293 cells. Without M3814 treatment, we found that XLF-L115A, XLF-4KA, and XLF- $\triangle \mathrm{KBM}$ were each able to promote No Indel EJ to the same degree as XLF-WT (Fig 5a). Thus, weakening the XLF-XRCC4 interface (L115A), or loss of the XLF-KU interface did not have an obvious effect on No Indel EJ. In contrast, XLF-L115D, which likely abolishes the XRCC4 interaction due to replacing a hydrophobic residue with a negative charge $^{30,31,38-41}$, showed a marked reduction in No Indel EJ, as described ${ }^{28}$. Next, when we examined each of these XLF mutants in combination with DNAPKcs kinase inhibition (M3814 treatment), we found that all of the XLF mutants caused a significant decrease in No Indel EJ compared to XLF-WT (Fig 5a).

We also examined the XLF mutants in the presence or absence of DNAPKcs using $X L F-K O / P R K D C-K O$ cells (Fig 5b). We found that in the absence of DNAPKcs, all the XLF mutants caused a significant decrease in No Indel EJ compared to XLF-WT. Furthermore, transient expression of DNAPKcs with all the XLF mutants caused a significant increase in No Indel EJ. For example, whereas in the $X L F-K O$ cell line the XLF$\triangle \mathrm{KBM}$ mutant showed no defect in No Indel EJ, in the $X L F-K O / P R K D C-K O$ cells this mutant was reduced 47-fold compared to XLF-WT, and transient expression of DNAPKcs was able to restore such EJ 25 -fold. These findings indicate that a series of XLF mutants that disrupt its interactions with XRCC4 and KU cause an enhanced requirement for DNAPKcs and its kinase activity for No Indel EJ. 
Given that a main phosphorylation target of DNAPKes appears to be itself at several sites, including the $\mathrm{ABCDE}$ and PQR clusters ${ }^{35,36,44}$, we posited that such autophosphorylation site clusters may be important to promote No Indel EJ in the context of a weakened XLF. To test this hypothesis, we examined No Indel EJ in XLFKO/PRKDC-KO HEK293 cells expressing XLF- $\triangle \mathrm{KBM}$ and XLF-K160D in combination with two DNAPKes mutants: the DNAPKes-ABCDE(S/T $>A)$ mutant that contains serine/threonine to alanine mutations at six phosphorylation sites (T2609, S2612, T2620, S2624, T2638, and T2647), and DNAPKcs-PQR(S $>A)$ mutant that contains such

mutations at five serine residues (S2023, S2029, S2041, S2053, and S2056) ${ }^{44,45}$. Beginning with $X L F-K O / P R K D C-K O$ HEK293 cells with or without XLF-WT, we found that similar to expressing DNAPKcs-WT, the mutants did not substantially affect No Indel EJ (Fig 5b, c). For $X L F-K O / P R K D C-K O$ HEK293 cells expressing XLF-K160D and XLF- $\triangle \mathrm{KBM}$, expression of DNAPKcs-WT markedly promotes No Indel EJ (Fig 5b), which enabled a functional examination of the DNAPKcs mutants. From this analysis we found that, when combined with either the XLF-K160D or XLF- $\triangle K$ KBM, DNAPKcs-PQR(S $>A)$ was not different from DNAPKcs-WT, whereas the DNAPKcs-ABCDE(S/T $>A)$ mutant showed a marked defect in No Indel EJ (Fig 5c). This finding indicates that the ABCDE phosphorylation sites cluster of DNAPKcs is critical for No Indel EJ when combined with a weakened XLF.

\section{DNAPKes kinase inhibition causes an increase in HDR and structural variants (SVs), and IR-sensitivity that is not further enhanced by XLF loss.}


Thus far our study has focused on the influence of the C-NHEJ factors DNAPKcs and XLF on EJ of blunt DSBs, but C-NHEJ also has other roles in DSB repair, such as suppression of homology-directed repair (HDR). Namely, loss of C-NHEJ factors (e.g., KU, DNAPKcs, and XRCC4), and inhibition of DNAPKes kinase activity, have been shown to cause an increase in $\mathrm{HDR}^{21,44,46,47}$. Thus, we sought to compare our above findings of the influence of DNAPKcs and XLF on blunt DSB EJ vs. suppression of HDR. To examine HDR, we used the LMNA-HDR assay, which measures HDR at the

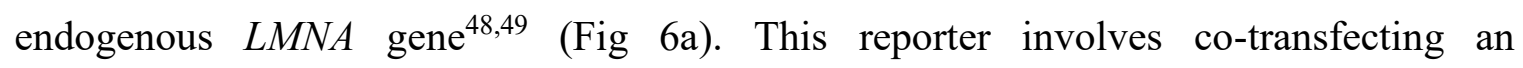
sgRNA/Cas9 expression vector to target a Cas9 DSB in $L M N A$ exon 1, along with a plasmid donor that contains the $L M N A$ exon 1 fused in frame with the fluorescent protein mRuby $2^{48,49}$. Thus, HDR using the plasmid donor causes mRuby $2+$ cells that can be detected via flow cytometry (Fig 6a) $)^{48,49}$. This assay has been validated to be dependent on the key HDR factor PALB2 ${ }^{48,49}$. We performed an independent validation using siRNA depletion of the HDR factor BRCA2 (siBRCA2) and found a marked reduction in mRuby2+ cells, compared to non-targeting siRNA (siCtrl, 12-fold, Fig 6b). As a comparison, we found that No Indel EJ was only modestly affected by siBRCA2 treatment (1.2-fold, Fig 6b).

Using this assay, we examined HDR frequencies in the PRKDC-KO,XLF-KO, and XRCC4-KO HEK293 cells, as well as parental cells treated with M3814. With each of these three KO cell lines, we found a significant increase in HDR, compared to the parental HEK293 cells (Fig 6c). In comparing the mutant lines, the PRKDC-KO cells showed significantly lower levels of HDR vs. $X L F-K O$, and $X R C C 4-K O$, however the relative differences were modest (Fig 6c). We then examined the effect of M3814 treatment in the 
parental cells, which we found significantly increased HDR with $250 \mathrm{nM}$, with no further increase with higher concentrations of M3814 (500 nM and $1000 \mathrm{nM}$, Fig 6d). This result indicates that the effect of M3814 on increasing HDR is saturated at $250 \mathrm{nM}$, which is notably different from the relatively mild effects of this concentration No Indel EJ, compared to $500 \mathrm{nM}$ and $1000 \mathrm{nM}$ (Fig 2a). Furthermore, the fold-increase of HDR caused by $500 \mathrm{nM} \mathrm{M} 3814$ in parental cells was similar to the effect of XLF loss, whereas No Indel EJ was only modestly affected by $500 \mathrm{nM} \mathrm{M} 3814$ vs. the marked defect in $X L F-K O$ cells (Fig 2a). Accordingly, we observe distinctions in the relative effect of M3814 on No Indel EJ vs. suppression of HDR.

We then sought to examine effects on HDR of combined disruption of DNAPKcs and XLF, as well as with the XLF-K160D mutant. Interestingly, we found that the XLF$K O / P R K D C-K O$ cells did not show an increase in HDR frequencies compared to the parental cells, and transient expression of XLF and DNAPKcs had no effect on HDR in $X L F-K O / P R K D C-K O$ cells (Supplemental Fig S3a), which we speculate may be due to an adaptation response in these cells to downregulate HDR. In any case, since the XLF and DNAPKcs expression vectors had no effect on HDR these cells, we were unable to examine mutants of these factors in this context. However, since XLF-WT expression suppressed HDR in the $X L F-K O$ cells (Fig 6c), we were able to examine XLF mutants alone, and in combination with M3814 treatment. From this analysis, we found that M3814 treatment in the $X L F-K O$ cells did not cause an increase in HDR (Fig 6d). From comparison of XLFWT vs. XLF-K160D in $X L F-K O$ cells, XLF-WT was significantly more proficient than XLF-K160D in suppressing HDR both in the absence of M3814 as well as at the lowest concentration $(250 \mathrm{nM})$, but with higher concentrations of M3814 (500 nM and $1000 \mathrm{nM})$, 
XLF-WT and XLF-K160D were not statistically different (Fig 6d). These findings indicate thar DNAPKcs kinase inhibition causes an increase in HDR that is not further enhanced by XLF loss.

Another reported outcome of sgRNA/Cas9 DSBs is large deletion mutations ${ }^{50,51}$, such that we sought to examine the influence of XLF and DNAPKcs kinase inhibition on such events. To study such deletions in human cells, we developed an assay using GFP fused to a protein degradation sequence $(\mathrm{GFPd} 2)^{52}$, which shortens its half-life, thereby enabling its use as a mutagenesis reporter ${ }^{53}$. We integrated GFPd2 into the FRT site of HEK293 cells, using the Flp recombinase, generated Cas9 DSBs using the PiggyBac system for stable expression of $\operatorname{sgRNAs} / \mathrm{Cas} 9^{50}$, and monitored the frequency of GFPnegative (GFP-neg cells). We induced DSBs at this locus in three positions: DSB-H that is $2.7 \mathrm{~kb}$ upstream from the start of the GFP cassette, DSB-L that is $0.41 \mathrm{~kb}$ downstream from the end of the GFP cassette, and DSB-G that is within the GFP coding sequence (Fig 6e). Each of these sgRNAs were confirmed to induce indels at the predicted target site (Supplemental Fig S3b). As compared to an sgRNA control that does not target the locus (No DSB), we found that each of these DSB sites induced GFP-neg cells, with DSB-G causing the highest frequency of GFP-neg cells, compared to DSB-H and DSB-L (Fig 6e). These findings indicate that DSBs far from the GFP expression cassette can induce GFPneg cells, which we then posited would be associated with loss of a substantial segment of the reporter locus. To test this hypothesis, we examined three sites in the reporter by quantitative PCR (qPCR): one downstream of DSB-H (site A), and two upstream from DSB-L (sites B and C) (Supplemental Fig S3b). We found that the GFP-neg cells induced by either DSB-H or DSB-L showed a significant decrease in amplicons from all three sites, 
compared to those induced by DSB-G that is within the GFP coding sequence (Supplemental Fig S3b). Thus, DSB-H and DSB-L induce GFP-neg events that are associated with loss of a substantial segment of the reporter locus. The most likely explanation for such events is a large deletion, as described ${ }^{50,51}$, however since more complex events could conceivably cause such genetic loss, we refer to them as structural variants (SVs), based on the definition of a genetic alteration involving $>50 \mathrm{bp}^{54}$. As such, we named this reporter GFPd2-SV.

Using this assay, we then examined the influence of XLF loss and M3814 treatment on the frequency of GFP-neg cells induced by DSBs at all three sites. Beginning with DSB$\mathrm{G}$ that is within the GFP coding sequence, M3814 treatment had no effect on the induction of GFP-neg cells, and XLF loss caused a modest decrease in the frequency of these events (Fig 6e). In contrast, for the DSBs induced away from the GFP expression cassette (DSBH and DSB-L), both M3814 treatment and XLF loss caused a significant increase in GFPneg cells (Fig 6e), indicating that DNAPKcs kinase activity and XLF are important to suppress DSB-induced SVs. We then compared the fold-increase in SVs, finding that 500 nM M3814 treatment had a similar effect as XLF loss, and 250 nM M3814 was only modestly lower than either (Fig 6e, both DSB-H and DSB-L). Furthermore, M3814 treatment had no effect on such SVs in the $X L F-K O$ cells (Fig 6e). Accordingly, similar to our findings with HDR, suppression of DSB-induced SVs is sensitive to inhibition of DNAPKcs kinase activity in a manner that is not further enhanced by loss of XLF.

Finally, we examined effects of XLF loss, XLF-K160D expression, and M3814 treatment on clonogenic survival following ionizing radiation (IR). First, we created $X L F$ KO HEK293 cell lines with stable expression of XLF-WT and XLF-K160D (Supplemental 
Fig S3c, control cell lines stably transfected with EV). As with the transient expression experiments, stable expression of XLF-K160D combined with M3814 treatment causes a marked reduction in No Indel EJ, compared to XLF-WT (Supplemental Fig S3c). Using these cell lines, we then examined fraction clonogenic survival following a 1 Gy IR, both with and without M3814 (500 nM). We chose 1 Gy IR for this analysis, because we found that this dose caused a marked reduction in clonogenic survival for both $X L F-K O$, as well as the parental line treated with M3814 (Fig 6f). We also found that addition of M3814 to $X L F-K O$ cells did not further enhance IR sensitivity (Fig 6f). Next, we examined IR treatment in $X L F-K O$ with stable expression of XLF-K160D and found a significant decrease compared to stable expression of XLF-WT (Fig 6f). Lastly, we examined combinations of M3814 treatment with both the XLF-WT and XLF-K160D cell lines, and found no significant difference in clonogenic survival after 1 Gy IR. These results are in accordance with the similar increase in HDR in M3814 treated XLF-WT and XLF-K160D (Fig 6d), but are distinct from the findings that combining XLF-K160D with M3814 causes a synergistic decrease in No Indel EJ (Fig 2a). Altogether, these findings indicate that suppression of HDR, SVs, and IR-resistance at 1 Gy are each sensitive to kinase inhibition of DNAPKcs in a manner that is not further enhanced by XLF loss. 


\section{DISCUSSION}

We sought to define the role of DNAPKcs on chromosomal DSB repair, with a focus on EJ of blunt DSBs, because other components of C-NHEJ (e.g., XLF and XRCC4) have been shown to be critical for such $\mathrm{EJ}^{30}$. To assess blunt DSB EJ, we examined two types of repair outcome: No Indel EJ between distal ends of two Cas9 blunt DSBs, as well as insertion mutations that are consistent with staggered Cas9 DSBs causing 5' overhangs that are filled-in to generate a blunt end. We found similar results with these two types of repair outcomes. Namely, DNAPKcs promotes these EJ events, but is less important than XLF, and its role is substantially magnified in the presence of a weakened XLF, i.e., XLFK160D, and other XLF mutations that disrupt its binding interfaces with XRCC4 and KU. Considering our findings in the context of recent cryo-EM structures ${ }^{8,9}$, we suggest that DNAPKcs is important to position a weakened XLF into an LR complex that can efficiently transition into an SR complex to mediate blunt DSB EJ, but that a weakened XLF cannot support a functional SR complex de novo (Fig 7). In contrast, with XLF-WT, the DNAPKcs LR complex does not appear to be absolutely required for establishing a functional SR complex for blunt DSB EJ. We speculate that the SR complex assembles de novo via a series of relatively weak protein-protein interactions, such that disrupting any of these interactions would abolish SR complex assembly unless first stabilized via DNAPKcs within the LR complex.

Based on these findings, the requirement for DNAPKcs during chromosomal EJ appears dependent on the specific circumstance. In Xenopus extracts, DNAPKcs is required for both establishing LR synapsis and transition to the SR synapsis state ${ }^{7}$. However, in the context of a chromosome in the cell nucleus, the stability of DSB end synapsis could be 
facilitated by higher-order chromosome structure ${ }^{55,56}$ that could provide some partial redundancy with the LR complex. Namely, in the context of chromatin, the SR complex may be proficient to form de novo and mediate blunt DSB EJ. Such functional redundancy for end synapsis may have evolved to ensure efficient blunt chromosomal DSB EJ ${ }^{56,57}$.

In addition to studies with DNAPKcs loss, we also found that DNAPKcs kinase inhibition, and the DNAPKcs-ABCDE(S/T>A) mutant, each show a marked reduction of blunt DSB EJ when combined with a weakened XLF. For example, from amplicon sequencing of EJ junctions, DNAPKcs kinase inhibition combined with XLF-K160D causes a marked reduction in blunt DSB EJ (No Indel EJ and insertions), and a converse increase in deletions. These findings are notable, because several lines of evidence indicate that DNAPKcs kinase inhibition and the DNAPKcs-ABCDE(S/T>A) mutant block DNAPKcs displacement from the LR complex. For one, the LR to SR transition is blocked by DNAPKcs kinase inhibitors in Xenopus extracts ${ }^{7}$. DNAPKcs kinase inhibitors have also been shown to cause a stable DNAPKcs complex on DSB ends that are prone to cleavage via the MRE11 nuclease ${ }^{34}$. As well, kinase-dead DNAPKcs shows persistent recruitment to laser DNA damage ${ }^{36}$. Finally, kinase-dead DNAPKcs causes severe defects in mouse embryonic development that is rescued with loss of $\mathrm{KU}^{58}$, which is also consistent with DNAPKcs kinase inhibition causing its hyper-stabilization on DNA ends. The likely mechanism of such stabilization is via blocking DNAPKcs trans-autophosphorylation. Specifically, mutation of such phosphorylation sites, particularly within the ABCDE cluster, causes persistent recruitment to laser DNA damage ${ }^{36}$, hyper-stable complexes on DNA ends with purified proteins ${ }^{59,60}$, and persistent association with damaged chromatin ${ }^{61}$. Altogether, we suggest that displacement of DNAPKcs is required for the LR complex to 
transition into a functional SR complex for blunt DSB EJ, which is particularly essential when combined with a weakened XLF that cannot support a functional SR complex de novo (Fig 7).

We also examined other roles of DNAPKes and XLF on DSB repair apart from blunt DSB EJ. For one, we examined regulation of HDR, which is known to be suppressed by C-NHEJ ${ }^{21,44,46,47}$. We found that suppression of HDR appears more sensitive to DNAPKcs kinase inhibition, compared to its effects on blunt DSB EJ. For example, a low dose of M3814 (250 nM) was saturating for causing an increase in HDR (vs. 500 and 1000 $\mathrm{nM}$ ), whereas these higher doses caused a concentration-dependent decrease in blunt DSB EJ. Furthermore, combining 500 nM M3814 with XLF loss causes a similar increase in HDR, compared to the M3814 treatment alone. In contrast, this combination of $500 \mathrm{nM}$ M3814 and XLF loss shows markedly lower blunt DSB EJ, compared to such M3814 treatment alone. We suggest a model whereby DNAPKcs kinase inhibition slows the transition from the LR to the SR complex, but such a delay may not have a marked effect on blunt DSB EJ, at least when combined with XLF-WT that could form a functional SR complex de novo. In contrast, such a delay could be sufficient to stimulate HDR, which likely involves initiation of DSB end resection that is mediated by the MRE11-RAD50NBS1 complex with $\mathrm{CtIP}^{34}$. Consistent with this notion, DNAPKcs kinase inhibition has been shown to promote MRE11-dependent cleavage of a DNAPKcs complex at DSB ends $^{34}$. Accordingly, the efficient/rapid transition of the LR complex to the SR complex may be critical for suppression of such end resection initiation.

One possible consequence of the deregulation of end resection initiation is genome instability. Namely, resected ends with long ssDNA are likely not readily repaired by C- 
NHEJ, nor HDR when the sister chromatid is unavailable. Thus, repair of such resected ends may be prone to more mutagenic processes. This model is consistent with our findings of DSB-induced SVs. Namely, similar to the findings with HDR, combining $500 \mathrm{nM}$ M3814 treatment with XLF loss caused a similar level of DSB-induced SVs, as compared to the M3814 treatment alone. Strikingly, we observed the same pattern with clonogenic survival following IR treatment. Accordingly, we speculate that DSB-induced SVs, along with other mutagenic events, could be a major contributing factor to the radiation sensitization caused by DNAPKcs kinase inhibition, rather than being due solely to a reduction in blunt DSB EJ. In conclusion, DNAPKcs appears critical for blunt DSB EJ when XLF is weakened, but also has distinct functions in regulating other aspects of genome maintenance. 


\section{METHODS}

\section{Plasmids and cell lines}

Sequences of sgRNAs and other oligonucleotides are in Supplemental Table S1. The px330 plasmid was used for sgRNA/Cas9 expression (Addgene 42230, generously deposited by Dr. Feng Zhang) ${ }^{1}$, except for the GFPd2-SV assay. Several plasmids were previously described: the EJ7-GFP reporter in the Pim1 mouse targeting vector, the sgRNA/Cas9 plasmids for the EJ7-GFP reporter (7a and 7b), the sgRNA/Cas9 plasmids for the GAPDH-CD4 assay (GAPDH and CD4), the pCAGGS-3xFLAG-XLF plasmids (both mouse and human), and pCAGGS-HA-XRCC4 ${ }^{28,30}$. Expression vectors for mutant forms of 3xFLAG-XLF were either previously described ${ }^{28,30,43}$, or generated via cloning in gBLOCKs and/or annealed oligonucleotides (Integrated DNA Technologies). The EJ7GFP +1 assay uses the $7 \mathrm{a}+1$ sgRNA instead of $7 \mathrm{a}$. The DNAPKes WT, ABCDE(S/T>A), and $\mathrm{PQR}(\mathrm{S}>\mathrm{A})$ expression plasmids were previously described (Addgene \#83317, \#83318 and $\# 83319$, respectively, generously deposited by Dr. Katheryn Meek $)^{44}$. Empty vector (EV) controls for XLF and XRCC4 used pCAGGS-BSKX, and for DNAPKcs used a CMV vector (pCMV6-XL5) ${ }^{62}$. The GFPd2-SV reporter was generated by introducing pCAGGFPd2 (Addgene \#14760, generously deposited by Dr. Connie Cepko) ${ }^{52}$ into pCDNA5/FRT (Thermofisher), and the sgRNAs for this reporter were DSB-H, DSB-G, and DSB-L, which were introduced into Piggybac gRNA-puro, which was generously provided by Dr. Allan Bradley (Wellcome Sanger Institute), along with the negative control sgRNA plasmid (\#5, No DSB), Piggybac Cas9-Blast plasmid, and Piggybac transposase expression plasmid ${ }^{50}$. The LMNA-HDR assay plasmids (LMNA Cas9/sgRNA, 
and LMNA-mRuby2-Donor) were previously described, and generously provided by Dr. Jean-Yves Masson (Laval University Cancer Research Center) ${ }^{49}$.

Several cell lines were described previously: HEK293 (EJ7-GFP parental line used to generate $X L F-K O$ and $X R C C 4-K O$ ), U2OS (EJ7-GFP parental and EJ7-GFP $X L F-K O$ ), and mESC EJ7-GFP reporter cell lines (WT, Xlf-/-, and Xrcc4-/-) ${ }^{28,30}$. The Prkdc-/- mESC line was generously provided by Dr. Frederick Alt (Harvard $)^{63}$. Cells were cultured as previously described ${ }^{28,30}$, and using the Lonza MycoAlert PLUS Mycoplasma Detection Kit, cell lines tested negative for mycoplasma contamination. Additional mutant cell lines were generated using Cas9/sgRNAs cloned into px330, as described previously ${ }^{28}$. Briefly, Cas9/sgRNA plasmids were co-transfected into cells either with a pgk-puro or dsRED plasmid, transfected cells were enriched using transient puromycin treatment or sorting for dsRED positive cells, followed by plating at low density to isolate and screen individual clones for gene disruption. The Prkdc-/- mESC line was used to generate the Prkdc-/-Xlf/- mESC using the sgRNAs mXlfsg1 and mXlfsg2 to create a deletion mutation, and the EJ7-GFP reporter was integrated into these cells by targeting to the Pim1 locus, as described $^{30}$. The HEK293 EJ7-GFP and U2OS EJ7-GFP cell lines were used to generate the PRKDC-KO cell lines with the sgRNAs PRKDCsg1 and PRKDCsg2 to create a deletion mutation, and the HEK293 EJ7-GFP PRKDC-KO cell line was used to generate the PRKDC-KO/XLF-KO cell line using the sgRNA XLFsg1. For the GFPd2-SV assay, the pCDNA5/FRT-GFPd2 plasmid was introduced into HEK293-FRT cells with pgkFLP as described ${ }^{62}$, and then used to generate an $X L F-K O$ cell line, using the sgRNA XLFsg1. The 3x-FLAG-XLF-WT and K160D stable expression cell lines were generated by cotransfection of the HEK293 EJ7-GFP XLF-KO cell line with these plasmids and pgk-puro, 
and selection for individual clones with puromycin that were subsequently screened. EV controls for these stable cell lines were generated by co-transfecting pCAGGS-BSKX and pgk-puro, and by pooling puromycin-resistant clones.

\section{DSB Reporter Assays}

For the EJ7-GFP and EJ7+1-GFP assays with HEK293 and U2OS cells, cells were seeded at $0.5 \times 10^{5}$ onto a 24 well, and transfected the following day with $200 \mathrm{ng}$ of each sgRNA/Cas9 plasmid (7a and 7b, or 7a+1 and 7b, respectively); 50 ng of XLF expression plasmid, XRCC4 expression plasmid, or control EV; and 200 ng of DNAPKcs expression plasmid or control EV, using $1.8 \mu \mathrm{L}$ of Lipofectamine 2000 (Thermofisher) in $0.5 \mathrm{ml}$ of antibiotic-free media. Transfection efficiency was determined with parallel wells that replace GFP expression vector and EV for the two sgRNA/Cas9 plasmids (i.e., $200 \mathrm{ng}$ pCAGGS-NZE-GFP and $200 \mathrm{ng}$ EV). For the LMNA-HDR assay, the LMNA Cas9/sgRNA and LMNA-mRuby2-Donor plasmids replace the two sgRNA/Cas9 plasmids (200 ng each). For mESC EJ7-GFP analysis, the plasmid amounts were $200 \mathrm{ng}$ each Cas9/sgRNA plasmids and 50 ng of XLF expression plasmid, XRCC4 expression plasmid, or EV. Cells were incubated with the transfection reagents for $4 \mathrm{hr}$, washed, and replaced with complete media, or for the M3814 analysis, with media containing M3814 (i.e., Nedisertib, MedChemExpress \#HY-101570 or Selleckchem \#S8586) and/or vehicle (Dimethyl Sulfoxide, DMSO) with all wells having the same total amount of DMSO in each experiment. Cells were analyzed 3 days after transfection using flow cytometry (Dako CyAN ADP, or ACEA Quanteon), as described ${ }^{30,64}$. For the GAPDH-CD4 analysis, the transfection conditions were the same, except using the GAPDH and CD4 Cas9/sgRNA plasmids, all amounts were scaled 4-fold to a 6 well dish, and cells stained with 
phycoerythrin-CD4 antibody (BioLegend, 317410) prior to analysis or isolation of CD4+ cells (BD Aria sorter), as described ${ }^{28}$. For all reporter assays, each bar represents the mean and error bars represent standard deviation, and the number of independent transfections and statistics are as described in the figure legends.

For the GFPd2-SV assay, HEK293-GFPd2-SV cells were transfected as for the other reporter assays, except using the plasmids 200ng Piggybac transposase, 200ng Piggybac Cas9-Blast, and 100ng of the respective Piggybac sgRNA-puro, although many of the experiments were scaled twofold to a 12 well. M3814 treatment was added as for the other reporter assays, and was continual through four days after transfection. The day after transfection, cells were treated with puromycin $(2 \mu \mathrm{g} / \mathrm{ml})$ and blasticidin $(7 \mu \mathrm{g} / \mathrm{ml})$ for a total of 9 days to select for cells with stable expression of $\operatorname{sgRNAs} / \mathrm{Cas} 9$, and allow for loss of GFPd2 protein. Cells were analyzed for GFP+ frequencies by flow cytometry as for the other reporter assays and/or used for cell sorting of GFP-negative (GFP-neg) cells (BD Aria sorter).

For GFPd2-SV reporter validation, samples sorted to enrich for GFP-neg cells were used for qPCR analysis (BioRad iTaq Universal SYBR Green Supermix \#1725120 and Biorad CFX96) with the primers described in Supplemental Table S1. Gemonic DNA was purified as described $^{64}$, digested overnight with BglII (New England Biolabs), and column purified (GFX / Illustra) prior to amplification. The Ct values for each locus within the reporter were subtracted from mean values for Actin control $(\Delta \mathrm{Ct})$, and then the mean $\Delta \mathrm{Ct}$ from the parental reporter cell line was subtracted $(\Delta \Delta \mathrm{Ct})$, and used to calculate locus amplification vs. the parental $\left(2^{-\Delta \Delta C t}\right)$. To validate the sgRNAs caused DSBs at the predicted site, genomic DNA from transfected cells were amplified with primers flanking 
each DSB site (Supplemental Table S1), and subjected to Tracking of Indels by Decomposition (i.e., TIDE) analysis ${ }^{65}$.

\section{GAPDH-CD4 rearrangement junction analysis}

CD4 + cells isolated following the DSB reporter assay described above were used to purify genomic DNA, as described, and the GAPDH-CD4 rearrangement junction was amplified with ILL-GAPDH and ILL-CD4 primers, which include the Illumina adapter sequences. The amplicons were subjected to deep sequencing using the Amplicon-EZ service (GENEWIZ), which includes their SNP/INDEL detection pipeline that aligned the reads to the No Indel EJ junction sequence as the reference sequence. The percentage of reads for the various indel types (No Indel, deletion, insertion, or complex indel) were quantified for each sample. Each cellular condition was examined with three independent transfections and $C D 4+$ sorted samples, the percentage of each indel type from the three samples was used to calculate the mean and standard deviation. Analysis of the insertion sequences was performed on all read sequences representing at least $0.1 \%$ of the total insertion reads.

\section{Clonogenic Survival Assays}

Cell lines were pre-treated with M3814 or vehicle (DMSO) for $3 \mathrm{hr}$ prior to trypsinization and resuspension in the same media as the pre-treatment, counted, and split into two groups: treatment with 1 Gy IR (Gammacell 3000) or left untreated. Cells were then plated in 6 well dishes at various cell densities in the same media as the pre-treatment with M3814 or vehicle (DMSO), and colonies allowed to form for 7-10 days, which were fixed in cold methanol prior to staining with $0.5 \%$ crystal violet (Sigma) in $25 \%$ methanol. 
Colonies were counted under a $4 \mathrm{X}$ objective and clonogenic survival was determined for each well relative to the mean value of DMSO/untreated wells for the respective cell line, with corrections for the plating density.

\section{Immunoblotting}

Cells were lysed with extraction with ELB (250 mM NaCl, 5 mM EDTA, $50 \mathrm{mM}$ Hepes, 0.1\% (v/v) Ipegal, and Roche protease inhibitor) with sonication (Qsonica, Q800R), or NETN Buffer (20 mM TRIS pH 8.0, 100 mM NaCl, 1 mM EDTA, 0.5\% IGEPAL, 1.25 mM DTT and Roche Protease Inhibitor) with several freeze/thaw cycles. For DNAPKcsS2056p analysis, cells were pre-treated with M3814 or vehicle (DMSO) for $3 \mathrm{hr}$, treated with 10 Gy IR (Gammacell 3000), allowed to recover for $1 \mathrm{hr}$, and protein extracted with ELB buffer containing PhosSTOP (Roche) and $50 \mu \mathrm{M}$ sodium fluoride. The transfections for immunoblotting analysis were identical to the reporter assays, except replacing EV (pCAGGS-BSKX) for the sgRNA/Cas9 plasmids, and scaled to a 6 well dish. Blots were probed with antibodies for DNAPKcs (Invitrogen MA5-13238), DNAPKcs-S2056p (Abcam ab124918), XLF (Bethyl A300-730A), XRCC4 (Santa Cruz sc271087), Tubulin (Sigma T9026), FLAG-HRP (Sigma A8592), ACTIN (Sigma A2066), HRP goat antimouse (Abcam ab205719), and HRP goat anti-rabbit (Abcam ab205718). ECL reagent (Amersham Biosciences) was used to develop HRP signals. DNAPKcs-S2056p signals were quantified with ImageJ. 


\section{ACKNOWLEDGEMENTS}

We thank Eva Jahanshir for technical support, and Dr. Jean-Yves Masson and Dr. Amélie Rodrigue (Laval University Cancer Research Center) for technical advice and reagents for the LMNA-HDR assay. This study was funded in part by the National Cancer Institute of the National Institutes of Health: R01CA256989, R01CA197506, R01CA240392 (J.M.S.); P30CA33572 (City of Hope Core Facilities).

\section{AUTHOR CONTRIBUTIONS}

M. C-A., L.J.T., and J.M.S. designed research and analyzed data; M. C-A., L.J.T.,

F.W.L., and R.B. performed research; M. C-A. and J.M.S. wrote the paper with input from all authors.

\section{COMPETING FINANCIAL INTEREST}

The authors have no competing financial interests to declare. 


\section{REFERENCES}

1. Ran, F.A. et al. Genome engineering using the CRISPR-Cas9 system. Nat Protoc 8, 2281-308 (2013).

2. Scully, R., Panday, A., Elango, R. \& Willis, N.A. DNA double-strand break repair-pathway choice in somatic mammalian cells. Nat Rev Mol Cell Biol 20, 698-714 (2019).

3. Ramsden, D.A. \& Nussenzweig, A. Mechanisms driving chromosomal translocations: lost in time and space. Oncogene 40, 4263-4270 (2021).

4. Iliakis, G. The role of DNA double strand breaks in ionizing radiation-induced killing of eukaryotic cells. Bioessays 13, 641-8 (1991).

5. Pannunzio, N.R., Li, S., Watanabe, G. \& Lieber, M.R. Non-homologous end joining often uses microhomology: implications for alternative end joining. $D N A$ repair 17, 74-80 (2014).

6. Mao, Z., Bozzella, M., Seluanov, A. \& Gorbunova, V. DNA repair by nonhomologous end joining and homologous recombination during cell cycle in human cells. Cell cycle 7, 2902-2906 (2008).

7. Graham, T.G., Walter, J.C. \& Loparo, J.J. Two-stage synapsis of DNA ends during non-homologous end joining. Molecular cell 61, 850-858 (2016).

8. Chen, S. et al. Structural basis of long-range to short-range synaptic transition in NHEJ. Nature, 593:294-298. (2021).

9. Chaplin, A.K. et al. Cryo-EM of NHEJ supercomplexes provides insights into DNA repair. Mol Cell 81, 3400-3409 e3 (2021).

10. Reid, D.A. et al. Organization and dynamics of the nonhomologous end-joining machinery during DNA double-strand break repair. Proc Natl Acad Sci U S A 112, E2575-84 (2015).

11. Zhao, B. et al. The essential elements for the noncovalent association of two DNA ends during NHEJ synapsis. Nature communications 10, 1-12 (2019).

12. Wang, X.S., Lee, B.J. \& Zha, S. The recent advances in non-homologous endjoining through the lens of lymphocyte development. DNA Repair (Amst) 94, 102874 (2020).

13. Corneo, B. et al. Rag mutations reveal robust alternative end joining. Nature 449, 483-6 (2007).

14. Taccioli, G.E. et al. Targeted disruption of the catalytic subunit of the DNA-PK gene in mice confers severe combined immunodeficiency and radiosensitivity. Immunity 9, 355-66 (1998).

15. Oksenych, V. et al. Functional redundancy between the XLF and DNA-PKcs DNA repair factors in $\mathrm{V}(\mathrm{D}) \mathrm{J}$ recombination and nonhomologous DNA end joining. Proc Natl Acad Sci US A 110, 2234-9 (2013).

16. Zha, S. et al. Ataxia telangiectasia-mutated protein and DNA-dependent protein kinase have complementary V(D)J recombination functions. Proc Natl Acad Sci $U S A$ 108, 2028-33 (2011).

17. Gapud, E.J. et al. Ataxia telangiectasia mutated (Atm) and DNA-PKcs kinases have overlapping activities during chromosomal signal joint formation. Proc Natl Acad Sci U S A 108, 2022-7 (2011). 
18. Liu, Y., Efimova, E.V., Ramamurthy, A. \& Kron, S.J. Repair-independent functions of DNA-PKcs protect irradiated cells from mitotic slippage and accelerated senescence. J Cell Sci 132, jcs229385 (2019).

19. Lu, H., Saha, J., Beckmann, P.J., Hendrickson, E.A. \& Davis, A.J. DNA-PKcs promotes chromatin decondensation to facilitate initiation of the DNA damage response. Nucleic Acids Res 47, 9467-9479 (2019).

20. Shao, Z. et al. DNA-PKcs has KU-dependent function in rRNA processing and haematopoiesis. Nature 579, 291-296 (2020).

21. Pierce, A.J., Hu, P., Han, M., Ellis, N. \& Jasin, M. Ku DNA end-binding protein modulates homologous repair of double-strand breaks in mammalian cells. Genes Dev 15, 3237-42 (2001).

22. Guirouilh-Barbat, J. et al. Impact of the KU80 pathway on NHEJ-induced genome rearrangements in mammalian cells. Mol Cell 14, 611-23 (2004).

23. Bennardo, N., Cheng, A., Huang, N. \& Stark, J.M. Alternative-NHEJ Is a Mechanistically Distinct Pathway of Mammalian Chromosome Break Repair. PLoS Genet 4, e1000110 (2008).

24. Bosma, G.C. et al. DNA-dependent protein kinase activity is not required for immunoglobulin class switching. J Exp Med 196, 1483-95 (2002).

25. Shou, J., Li, J., Liu, Y. \& Wu, Q. Precise and Predictable CRISPR Chromosomal Rearrangements Reveal Principles of Cas9-Mediated Nucleotide Insertion. Mol Cell 71, 498-509 e4 (2018).

26. Jiang, F. \& Doudna, J.A. CRISPR-Cas9 Structures and Mechanisms. Annu Rev Biophys 46, 505-529 (2017).

27. Geisinger, J.M., Turan, S., Hernandez, S., Spector, L.P. \& Calos, M.P. In vivo blunt-end cloning through CRISPR/Cas9-facilitated non-homologous end-joining. Nucleic Acids Res 44, e76 (2016).

28. Bhargava, R., Lopezcolorado, F.W., Tsai, L.J. \& Stark, J.M. The canonical nonhomologous end joining factor XLF promotes chromosomal deletion rearrangements in human cells. J Biol Chem 295, 125-137 (2020).

29. Olivieri, M. et al. A Genetic Map of the Response to DNA Damage in Human Cells. Cell 182, 481-496 e21 (2020).

30. Bhargava, R. et al. C-NHEJ without indels is robust and requires synergistic function of distinct XLF domains. Nature communications 9, 1-14 (2018).

31. Andres, S.N., Modesti, M., Tsai, C.J., Chu, G. \& Junop, M.S. Crystal structure of human XLF: a twist in nonhomologous DNA end-joining. Molecular cell 28, 1093-1101 (2007).

32. DeFazio, L.G., Stansel, R.M., Griffith, J.D. \& Chu, G. Synapsis of DNA ends by DNA-dependent protein kinase. Embo j 21, 3192-200 (2002).

33. Li, Y. et al. Crystal structure of human XLF/Cernunnos reveals unexpected differences from XRCC4 with implications for NHEJ. The EMBO journal 27, 290-300 (2008).

34. Deshpande, R.A. et al. DNA-dependent protein kinase promotes DNA end processing by MRN and CtIP. Sci Adv 6, eaay0922 (2020).

35. Meek, K., Douglas, P., Cui, X., Ding, Q. \& Lees-Miller, S.P. trans Autophosphorylation at DNA-dependent protein kinase's two major 
autophosphorylation site clusters facilitates end processing but not end joining. Molecular and cellular biology 27, 3881-3890 (2007).

36. Uematsu, N. et al. Autophosphorylation of DNA-PKCS regulates its dynamics at DNA double-strand breaks. Journal of Cell Biology 177, 219-229 (2007).

37. Zenke, F.T. et al. Pharmacologic inhibitor of DNA-PK, M3814, potentiates radiotherapy and regresses human tumors in mouse models. Molecular cancer therapeutics 19, 1091-1101 (2020).

38. Graham, T.G.W., Carney, S.M., Walter, J.C. \& Loparo, J.J. A single XLF dimer bridges DNA ends during nonhomologous end joining. Nat Struct Mol Biol 25, 877-884 (2018).

39. Mahaney, B.L., Hammel, M., Meek, K., Tainer, J.A. \& Lees-Miller, S.P. XRCC4 and XLF form long helical protein filaments suitable for DNA end protection and alignment to facilitate DNA double strand break repair. Biochemistry and Cell Biology 91, 31-41 (2013).

40. Malivert, L. et al. Delineation of the Xrcc4-interacting region in the globular head domain of cernunnos/XLF. J Biol Chem 285, 26475-83 (2010).

41. Normanno, D. et al. Mutational phospho-mimicry reveals a regulatory role for the XRCC4 and XLF C-terminal tails in modulating DNA bridging during classical non-homologous end joining. Elife 6, e22900 (2017).

42. Hammel, M. et al. XRCC4 protein interactions with XRCC4-like factor (XLF) create an extended grooved scaffold for DNA ligation and double strand break repair. J Biol Chem 286, 32638-50 (2011).

43. Carney, S.M. et al. XLF acts as a flexible connector during non-homologous end joining. Elife 9, e61920 (2020).

44. Cui, X. et al. Autophosphorylation of DNA-dependent protein kinase regulates DNA end processing and may also alter double-strand break repair pathway choice. Molecular and cellular biology 25, 10842-10852 (2005).

45. Goodarzi, A.A. et al. DNA-PK autophosphorylation facilitates Artemis endonuclease activity. The EMBO journal 25, 3880-3889 (2006).

46. Robert, F., Barbeau, M., Ethier, S., Dostie, J. \& Pelletier, J. Pharmacological inhibition of DNA-PK stimulates Cas9-mediated genome editing. Genome Med 7, 93 (2015).

47. Shrivastav, M. et al. DNA-PKcs and ATM co-regulate DNA double-strand break repair. DNA Repair (Amst) 8, 920-9 (2009).

48. Boonen, R. et al. Functional analysis of genetic variants in the high-risk breast cancer susceptibility gene PALB2. Nat Commun 10, 5296 (2019).

49. Rodrigue, A. et al. A global functional analysis of missense mutations reveals two major hotspots in the PALB2 tumor suppressor. Nucleic acids research 47, 10662-10677 (2019).

50. Kosicki, M., Tomberg, K. \& Bradley, A. Repair of double-strand breaks induced by CRISPR-Cas9 leads to large deletions and complex rearrangements. Nat Biotechnol 36, 765-771 (2018).

51. Roidos, P. et al. A scalable CRISPR/Cas9-based fluorescent reporter assay to study DNA double-strand break repair choice. Nat Commun 11, 4077 (2020).

52. Matsuda, T. \& Cepko, C.L. Controlled expression of transgenes introduced by in vivo electroporation. Proc Natl Acad Sci U S A 104, 1027-32 (2007). 
53. $\mathrm{Fu}, \mathrm{Y}$. et al. High-frequency off-target mutagenesis induced by CRISPR-Cas nucleases in human cells. Nat Biotechnol 31, 822-6 (2013).

54. Carvalho, C.M. \& Lupski, J.R. Mechanisms underlying structural variant formation in genomic disorders. Nat Rev Genet 17, 224-38 (2016).

55. Clouaire, T. \& Legube, G. A Snapshot on the Cis Chromatin Response to DNA Double-Strand Breaks. Trends Genet 35, 330-345 (2019).

56. Stinson, B.M. \& Loparo, J.J. Repair of DNA Double-Strand Breaks by the Nonhomologous End Joining Pathway. Annu Rev Biochem 90, 137-164 (2021).

57. Chang, H.H., Pannunzio, N.R., Adachi, N. \& Lieber, M.R. Non-homologous DNA end joining and alternative pathways to double-strand break repair. Nature reviews Molecular cell biology 18, 495-506 (2017).

58. Jiang, W. et al. Differential phosphorylation of DNA-PKcs regulates the interplay between end-processing and end-ligation during nonhomologous end-joining. Mol Cell 58, 172-85 (2015).

59. Block, W.D. et al. Autophosphorylation-dependent remodeling of the DNAdependent protein kinase catalytic subunit regulates ligation of DNA ends. Nucleic Acids Res 32, 4351-7 (2004).

60. Reddy, Y.V., Ding, Q., Lees-Miller, S.P., Meek, K. \& Ramsden, D.A. Nonhomologous end joining requires that the DNA-PK complex undergo an autophosphorylation-dependent rearrangement at DNA ends. J Biol Chem 279, 39408-13 (2004).

61. Neal, J.A. et al. Unraveling the complexities of DNA-dependent protein kinase autophosphorylation. Mol Cell Biol 34, 2162-75 (2014).

62. Kelso, A.A., Lopezcolorado, F.W., Bhargava, R. \& Stark, J.M. Distinct roles of RAD52 and POLQ in chromosomal break repair and replication stress response. PLoS Genet 15, e1008319 (2019).

63. Gao, Y. et al. A Targeted DNA-PKcs-Null Mutation Reveals DNA-PKIndependent Functions for KU in V(D)J Recombination. Immunity 9, 367-376 (1998).

64. Gunn, A. \& Stark, J.M. I-SceI-based assays to examine distinct repair outcomes of mammalian chromosomal double strand breaks. Methods Mol Biol 920, 379-91 (2012).

65. Brinkman, E.K., Chen, T., Amendola, M. \& van Steensel, B. Easy quantitative assessment of genome editing by sequence trace decomposition. Nucleic Acids Res 42, e168 (2014). 


\section{FIGURE LEGENDS}

Figure 1. DNAPKes is less important for No Indel EJ vs. XLF and XRCC4, but becomes essential in combination with an XLF mutant (K160D). (a) Shown is the EJ7GFP reporter for No Indel EJ (not to scale), which is chromosomally integrated using the Flp-FRT system in HEK293 and U2OS cells, and targeting to the Pim1 locus of mESCs. Cells are transfected with expression vectors for Cas9 and the two sgRNAs, with complementing vector(s) or empty vector (EV), and GFP frequencies are normalized to transfection efficiency with parallel GFP transfections. (b) DNAPKcs is less important for No Indel EJ compared to XLF and XRCC4 in HEK293 cells. $n=6$. Statistics with unpaired $t$-test using Holm-Sidak correction. Immunoblots show levels of DNAPKcs and XRCC4. (c) A reported structure of the XLF homodimer (aa 1-227, Protein Data Bank 2R9A), with the K160/D161 salt-bridge highlighted. (d) DNAPKcs is required for No Indel EJ in HEK293 cells with XLF-K160D. $n=6$. Statistics as in (b). Immunoblots show levels of DNAPKcs, XLF-WT, and XLF-K160D. (e) The influence of DNAPKcs on No Indel EJ is conserved in mESCs. $n=6$. Statistics as in (b). Immunoblots show levels of XLF-WT and XLF-K160D. $* * * * P<0.0001, * P<0.05, \dagger P<0.05$ but not significant after correction (unadjusted $P$-value). Error bars $=\mathrm{SD}$.

Figure 2: Inhibiting DNAPKes kinase activity suppresses No Indel EJ to a greater degree when combined with XLF-K160D. (a) Effect of DNAPKcs kinase inhibitor M3814 on No Indel EJ in HEK293 cells with XLF-WT, XLF-K160D, and without XLF. Control cells treated with DMSO (the vehicle for M3814). $\mathrm{n}=6$. Statistics with unpaired $t$ test using Holm-Sidak correction. (b) M3814 decreases ionizing radiation (IR)-induced phosphorylation of DNAPKcs at S2056 (S2056p). Shown are levels of DNAPKcs-S2056p 
normalized to Actin, and a representative immunoblot. $n=4$. Statistics as in (a). (c) The influence of DNAPKcs kinase inhibition with XLF-K160D is similar in U2OS cells. $n=6$. Statistics as in (a). ${ }^{* * * *} P<0.0001, * * P<0.01, * P<0.05, \dagger P<0.05$ but not significant after correction (unadjusted $P$-value), n.s. $=$ not significant. Error bars $=\mathrm{SD}$.

\section{Figure 3: Inhibiting DNAPKes kinase activity affects EJ junction patterns to a greater} degree when combined with XLF-K160D. (a) Shown is the GAPDH-CD4 rearrangement assay, which uses the endogenous genes (not to scale). HEK293 cells are transfected with expression vectors for Cas9 and the two sgRNAs, with complementing vector(s) or empty vector $(\mathrm{EV})$. CD4 frequencies are normalized to transfectionn efficiency with parallel GFP transfections. CD4+ cells are sorted using FACS and used to analyze the GAPDH-CD4 junction patterns by amplicon deep sequencing. (b) Loss of DNAPKcs and XLF leads to decreased CD4+ cells. HEK293 cells were treated with DMSO or 500 nM M3814. n=6. Statistics with unpaired $t$-test using Holm-Sidak correction. (c) Combining 500 nM M3814 and XLF-K160D causes a decrease in $C D 4+$ cells. $\mathrm{n}=6$. Statistics as in (b). (d) DNAPKcs is less important to promote No Indel EJ and insertions and suppress deletions compared to XLF. Shown are four types of EJ junctions from amplicon deep sequencing. $n=3$. Statistics as in (b). (e) DNAPKcs kinase inhibition affects EJ junctions to a greater degree when combined with XLF-K160D. The frequency of the four types of EJ junctions in (d) are shown. $\mathrm{n}=3$. Statistics as in (b). $* * * * P<0.0001, * * * \mathrm{P}<0.001, * * P<0.01, * P<0.05$, $\dagger P<0.05$ but not significant after correction (unadjusted $P$-value), n.s. $=$ not significant. Error bars $=$ SD.

Figure 4: EJ with insertions that are likely caused by staggered Cas9 DSBs show similar genetic requirements as No Indel EJ. (a) Shown is the EJ7+1-GFP reporter (not 
to scale) for EJ with $1 \mathrm{nt}$ insertions (+1 EJ), which is the same chromosomal reporter as in Figure 1, but using a different 5' sgRNA. (b) DNAPKcs is less important to promote +1 EJ compared to XLF. $\mathrm{n}=6$. Statistics with unpaired $t$-test using Holm-Sidak correction. (c) DNAPKcs is required for +1 EJ in cells with XLF-K160D. $n=6$. Statistics as in (b). (d) Inhibiting DNAPKcs kinase activity has a markedly greater effect on $+1 \mathrm{EJ}$ when combined with XLF-K160D. $\mathrm{n}=6$. Statistics as in (b). $* * * * P<0.0001, * * * \mathrm{P}<0.001$, n.s. $=$ not significant. Error bars $=\mathrm{SD}$.

Figure 5: DNAPKes becomes essential for No Indel EJ in combination with other mutants in XLF binding interfaces, and depends on the DNAPKcs-ABCDE phosphorylation sites cluster. (a) DNAPKcs kinase inhibition has a markedly greater effect on No Indel EJ in cells with XLF mutants disrupting the XRCC4 or KU interaction interfaces. $\mathrm{n}=6$. Statistics with unpaired $t$-test using Holm-Sidak correction. Also shown is the XLF homodimer (aa 1-227, Protein Data Bank 2R9A, with the C-terminus for one monomer drawn as a line), with the L115 residue important for XRCC4 interaction, $\mathrm{Ku}$ Binding Motif (KBM), and 4 lysine $(\mathrm{K})$ residues in the $\mathrm{KBM}$ highlighted. Immunoblot shows levels of FLAG-tagged XLF-WT and mutants. (b) DNAPKcs is required to promote No Indel EJ in cells with XLF mutants disrupting the XRCC4 and KU interaction interfaces. Statistics as in (a). Immunoblots show levels of FLAG-tagged XLF-WT and mutants, and DNAPKcs. (c) DNAPKcs-ABCDE phosphorylation cluster is required to promote No Indel EJ in cells expressing XLF- $\triangle$ KBM and XLF-K160D. n=6. Statistics as in (a). Immunoblot shows levels of DNAPKcs-WT, DNAPKcs-ABCDE(S/T>A), and DNAPKcs-PQR(S $>$ A). $* * * * P<0.0001, \quad * * * \mathrm{P}<0.001, * * P<0.01, * P<0.05$, n.s. $=$ not significant. Error bars $=\mathrm{SD}$. 
Figure 6: DNAPKes kinase inhibition causes an increase in HDR and structural variants (SVs), and IR-sensitivity that is not further enhanced by XLF loss. (a) Shown is the LMNA-HDR reporter (not to scale) involving a Cas9/sgRNA that induces a DSB in $L M N A$ exon 1, and a plasmid donor such that HDR causes mRuby2 to be expressed from the $L M N A$ locus. The mRuby2 frequencies are normalized to transfection efficiency with parallel GFP transfections. (b) BRCA2 is required for HDR by the LMNA-HDR assay, but has modest effects on No Indel EJ. Cells were transfected with siRNA that is non-targeting ( $\operatorname{siCtrl})$ or targets BRCA2 (siBRCA2). $\mathrm{n}=6$. Statistics with unpaired $t$-test using HolmSidak correction. Immunoblot shows levels of BRCA2. (c) DNAPKcs, XLF, and XRCC4 suppress HDR to similar degrees. $n=6$. Statistics as in (b). (d) Suppression of HDR. $n=6$. Statistics as in (b). (e) Suppression of SVs. Shown is the GFPd2-SV reporter (not to scale) that is chromosomally integrated to an FRT site in HEK293 cells. Stable expression of Cas9 and various sgRNAs (DSB-H, DSB-G, and DSB-L) can induce GFP-negative cells (GFP-neg). n=6. Statistics as in (b). (f) IR-sensitivity. HEK293 stable cell lines were treated with DMSO or M3814, and 0 Gy or 1 Gy IR dose, and plated to form colonies. Fraction clonogenic survival was determined relative to DMSO 0 Gy for each cell line. $\mathrm{n}=6$. Statistics as in (b). $* * * * P<0.0001, * * * \mathrm{P}<0.001, * * P<0.01, * P<0.05, \dagger P<0.05$ but not significant after correction (unadjusted $P$-value), n.s. $=$ not significant. Error bars $=\mathrm{SD}$.

Figure 7. Summary. Model for the DNAPKcs LR C-NHEJ complex being important to stabilize a weakened XLF to transition into a functional SR complex to facilitate blunt DSB EJ. Illustrations adapted from published cryo-EM structures described in the text. Light green checkmark depicts a partial defect, whereas a red X denotes a marked loss of blunt DSB EJ. 
a

\section{EJ7-GFP}

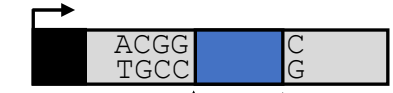

Cas9/sgRnA $\uparrow$ of

Two Blunt DSB $\downarrow$

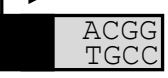

No Indel EJ $\downarrow$

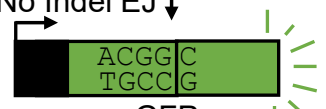

C

d

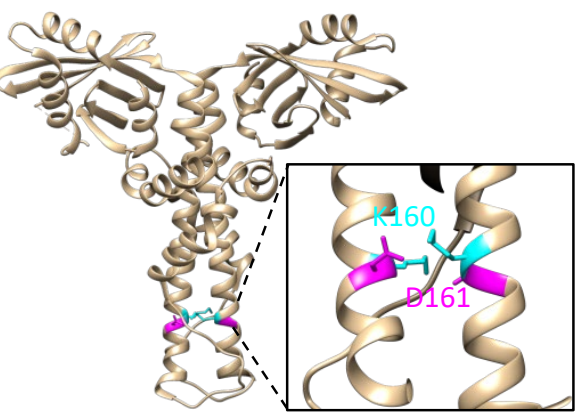

HEK293

$\mathrm{x}=$ fold vs. XLF-WT (in XLF-KO)

DNAPKcs EV EV EV WT EV EV WT EV EV WT

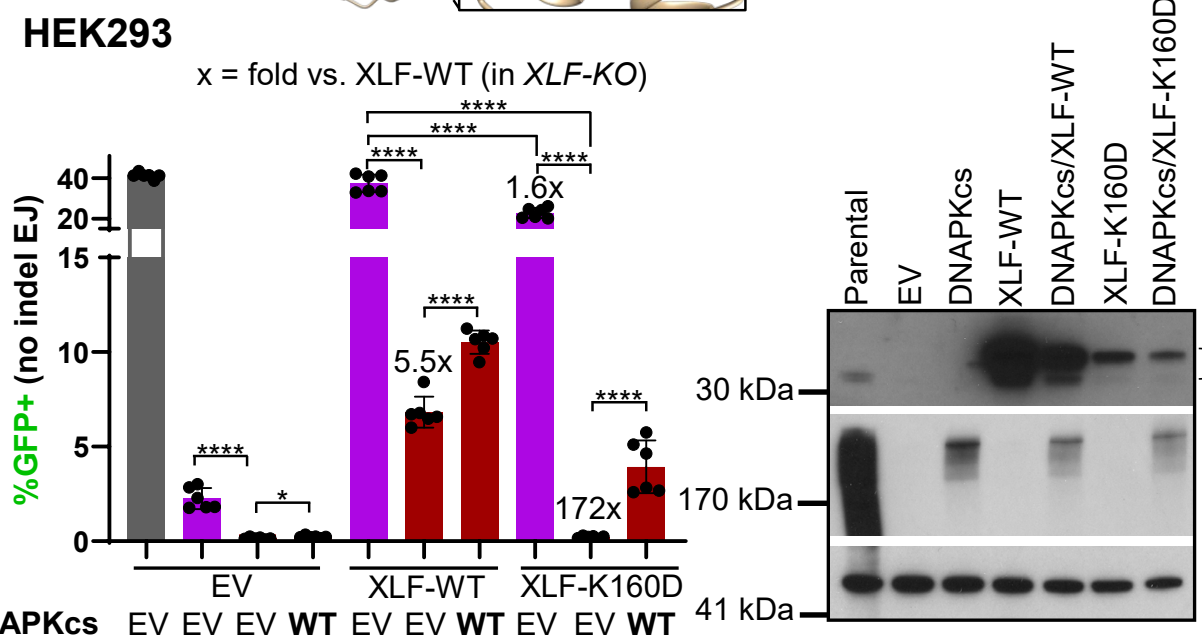

b HEK293

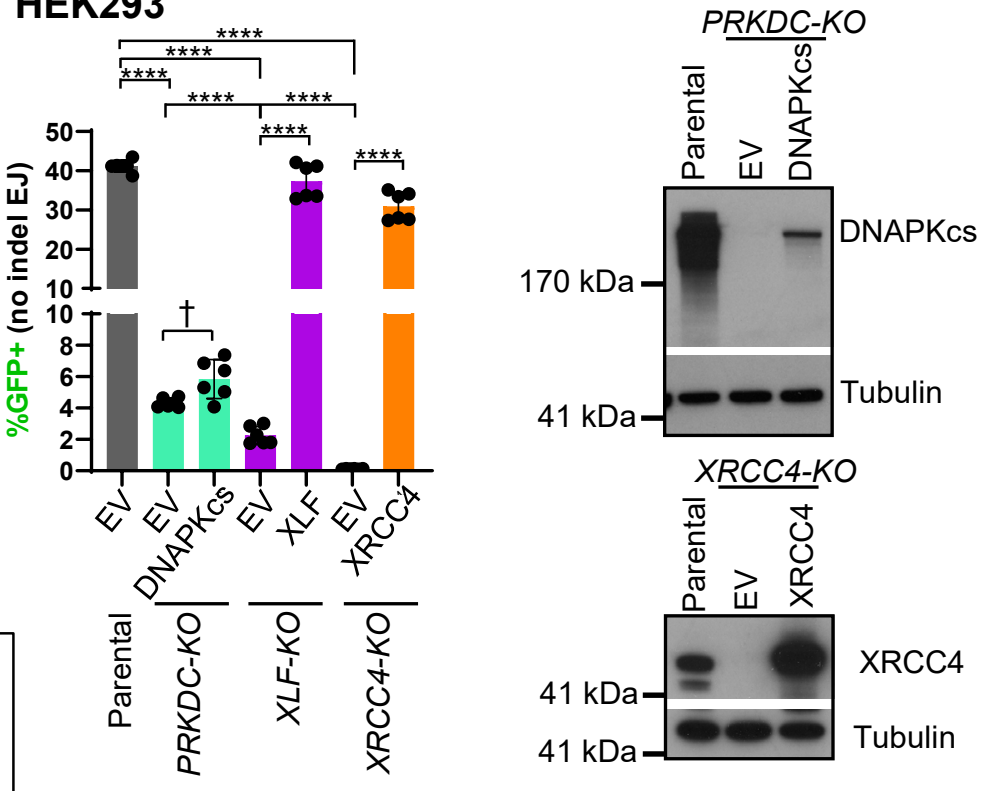

Cisneros-Aguirre et. al. Figure 1

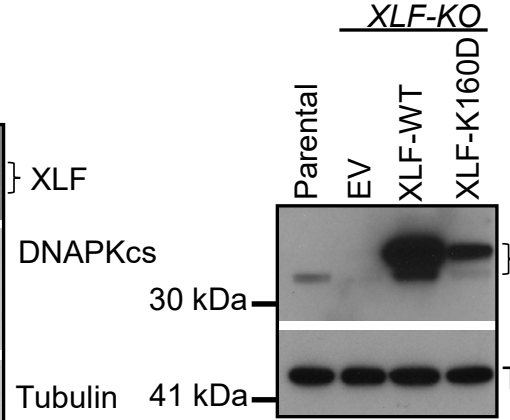

$\mathrm{XLF}$

e

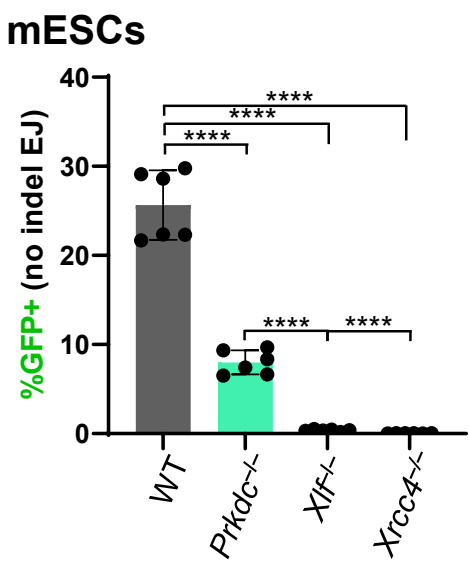

mESCs

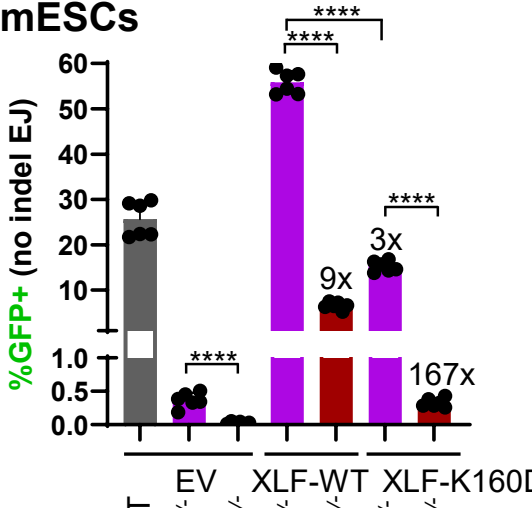

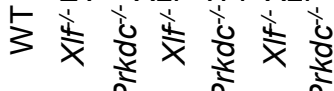

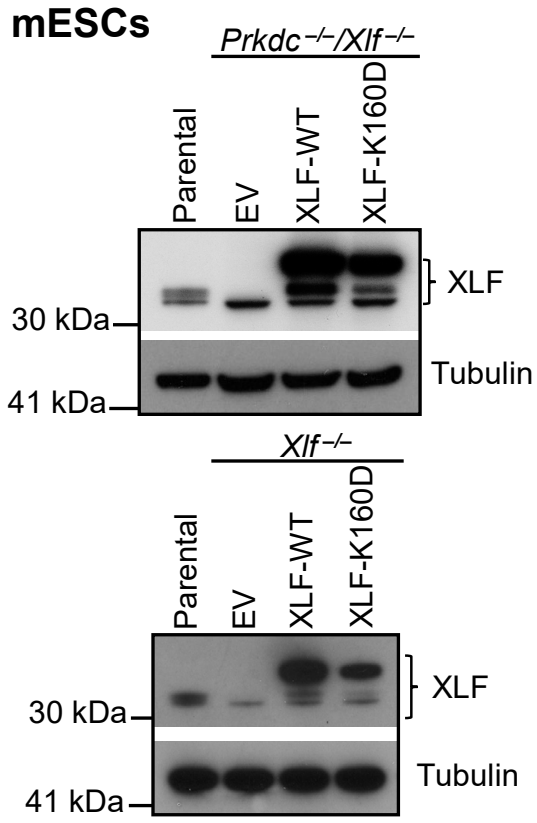




\section{HEK293}

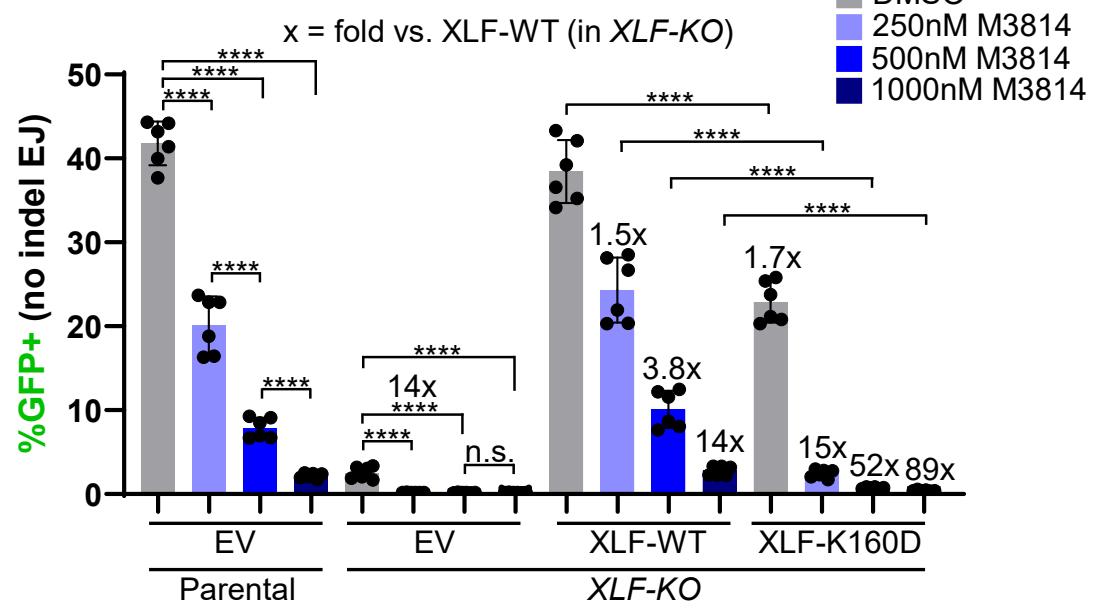

C

\section{U2OS}

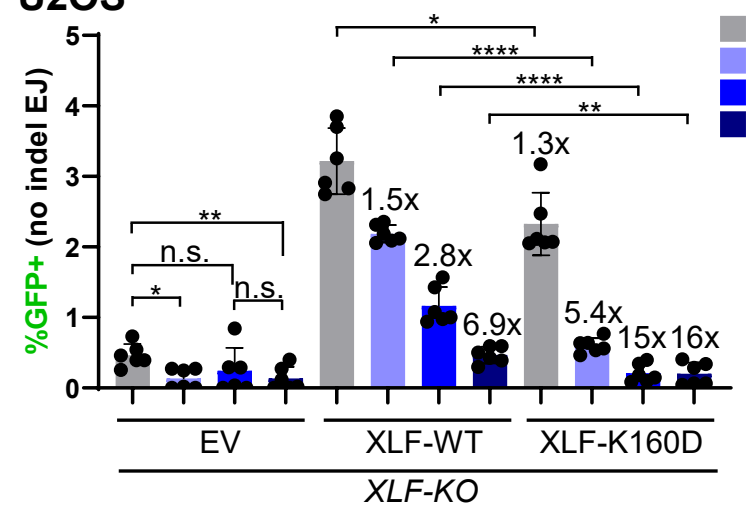

DMSO

250nM M3814

500nM M3814
1000nM M3814 b

\section{HEK293}
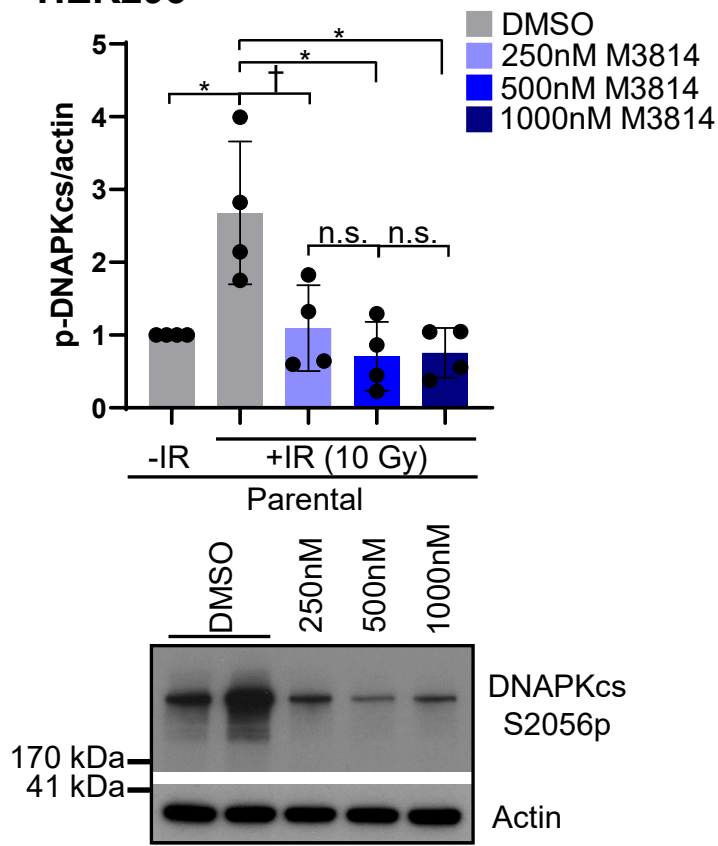

Cisneros-Aguirre et. al. Figure 2

$170 \mathrm{kDa}$

Actin 
a

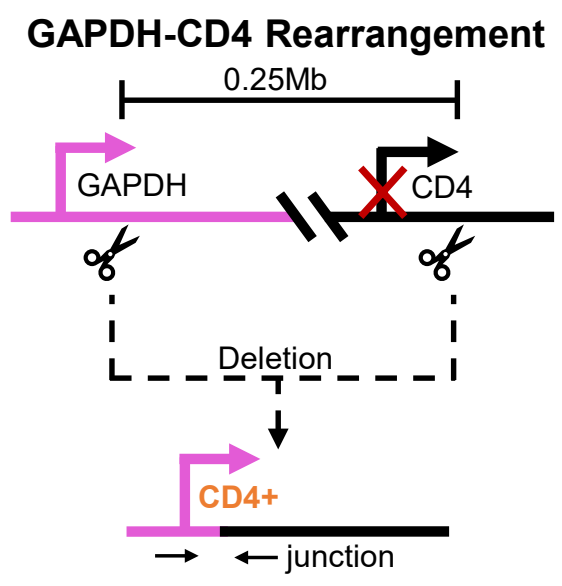

C

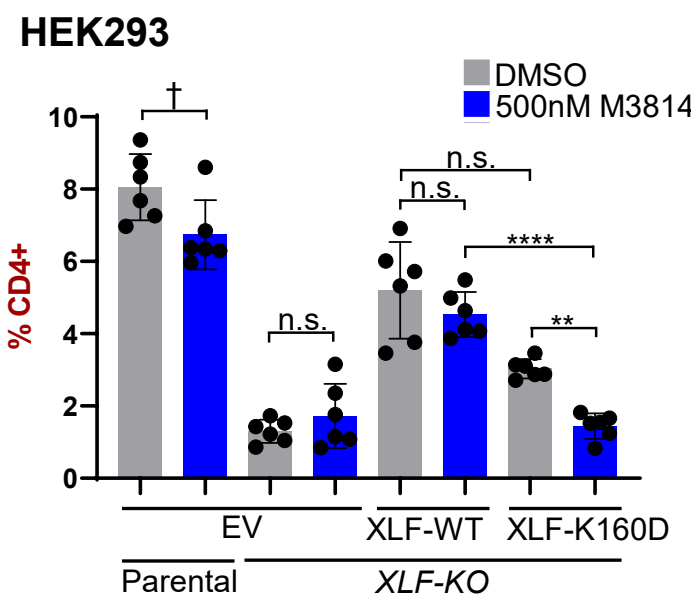

e
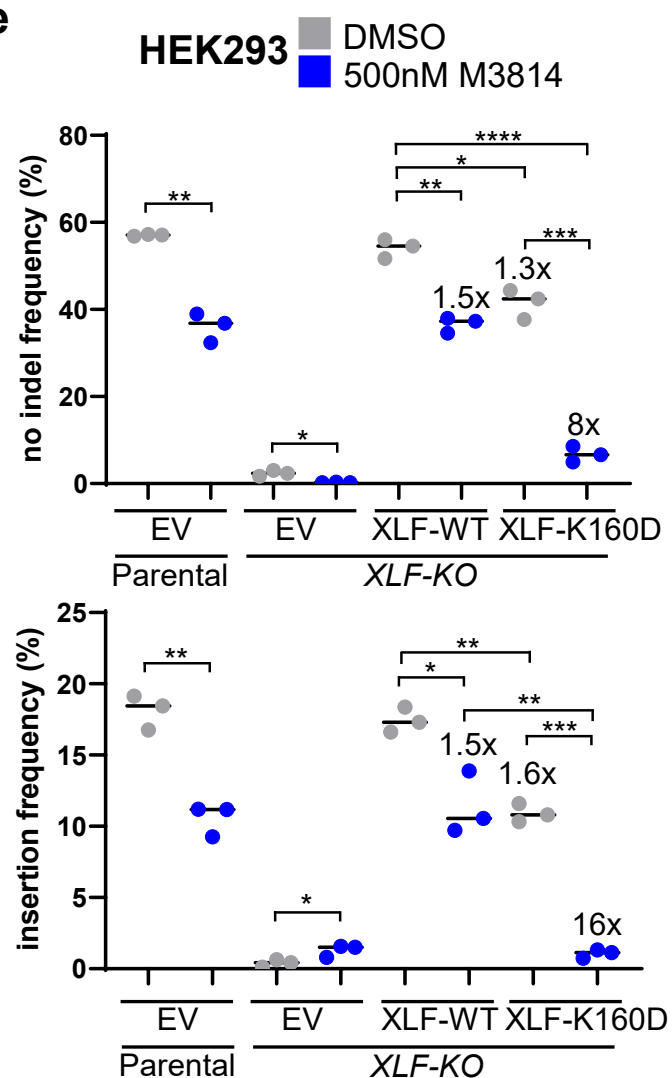

b

Cisneros-Aguirre et. al. Figure 3

\section{HEK293}

d

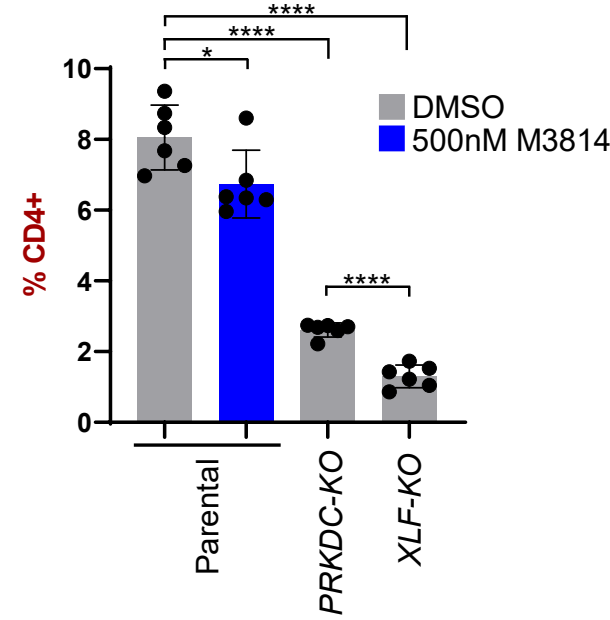

\section{HEK293}

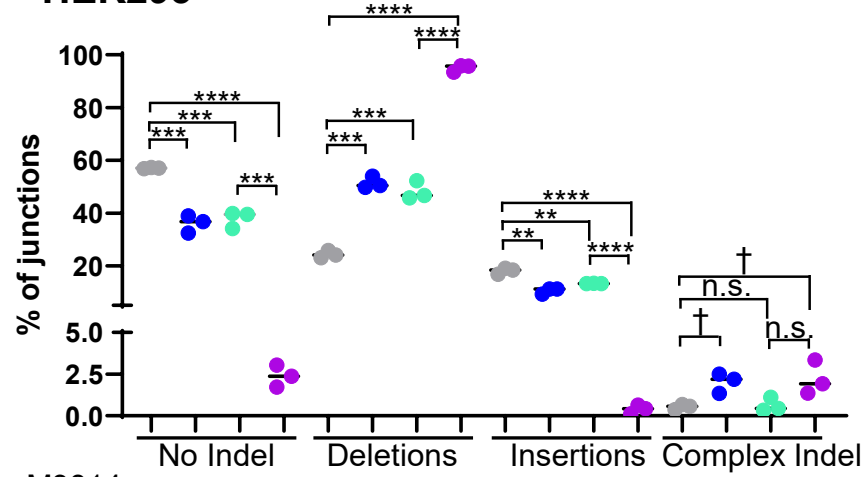

M3814
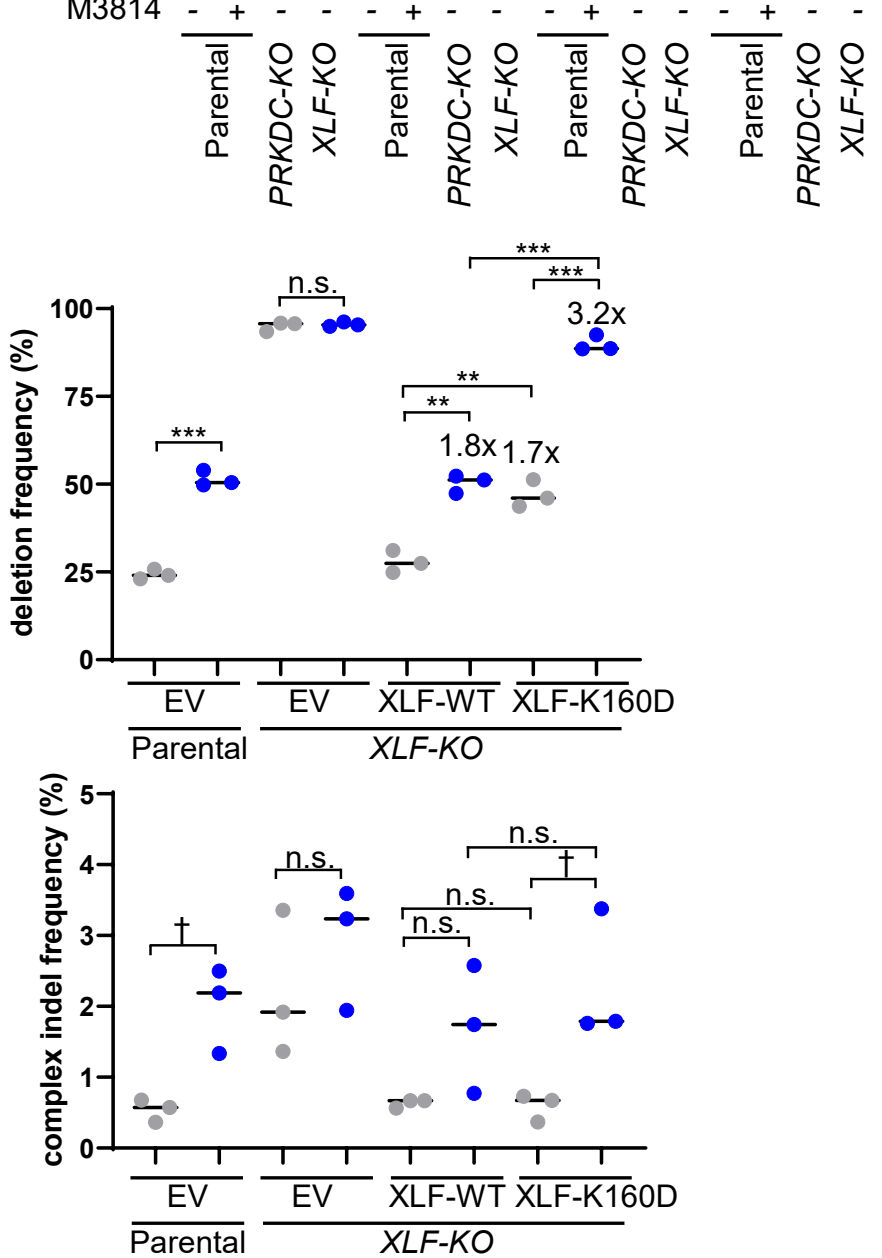
a

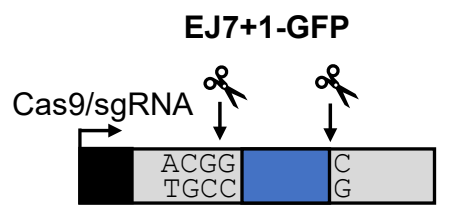

5' overhang DSB $\downarrow$

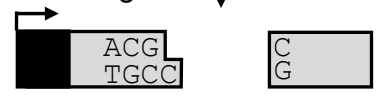

$1 \mathrm{nt}$ synthesis $\downarrow$
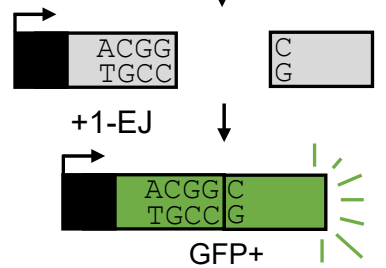

C

\section{HEK293}

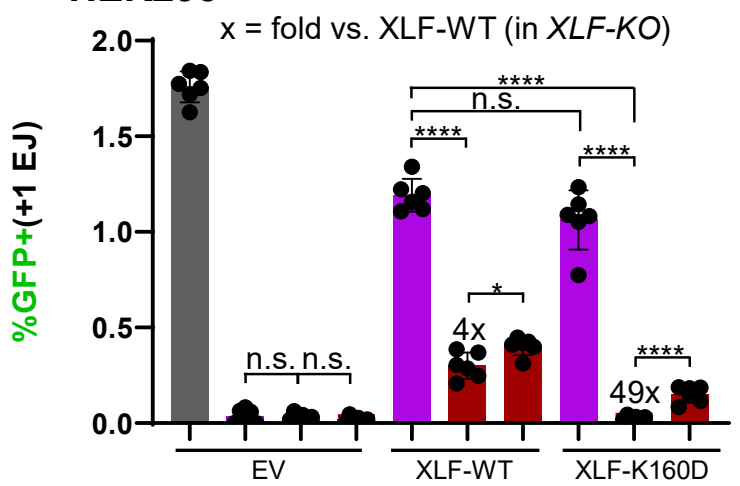

DNAPKcs

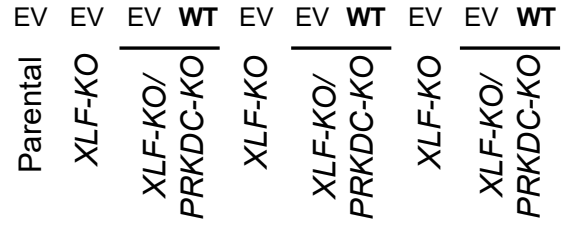

b

HEK293

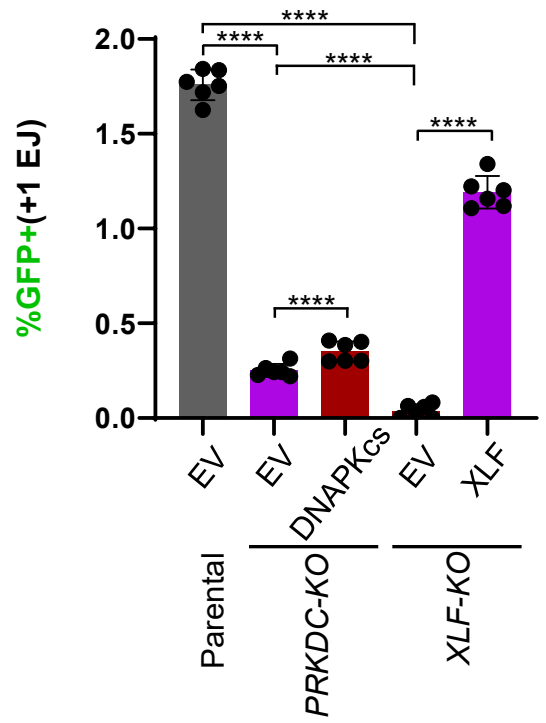

d

HEK293

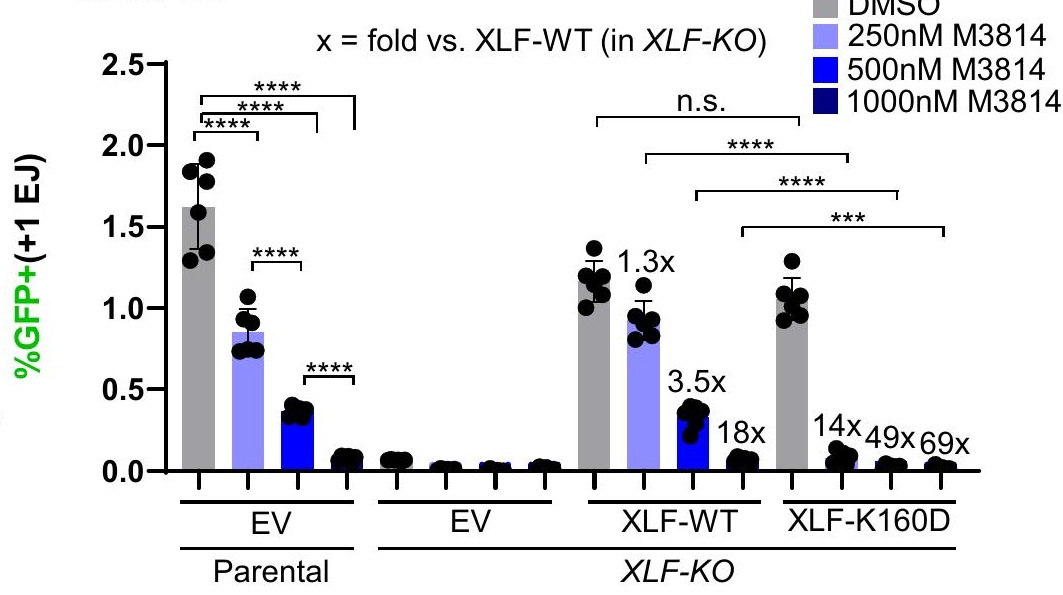




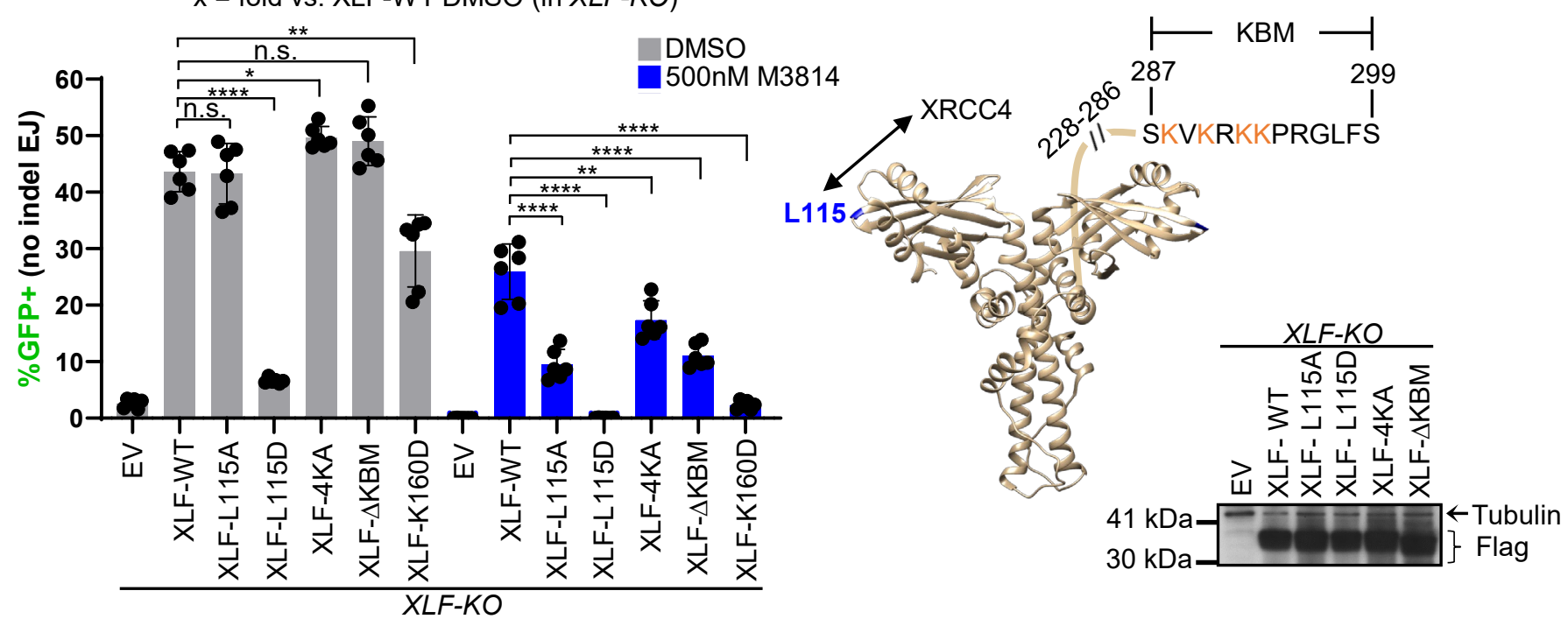

b
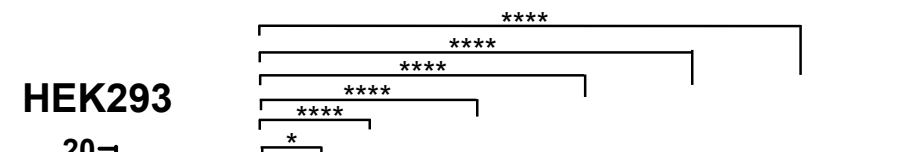

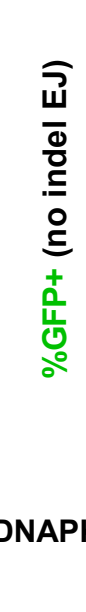

\section{HEK293}

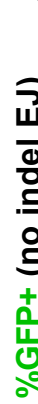

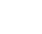

$$
\text { DN }
$$

C

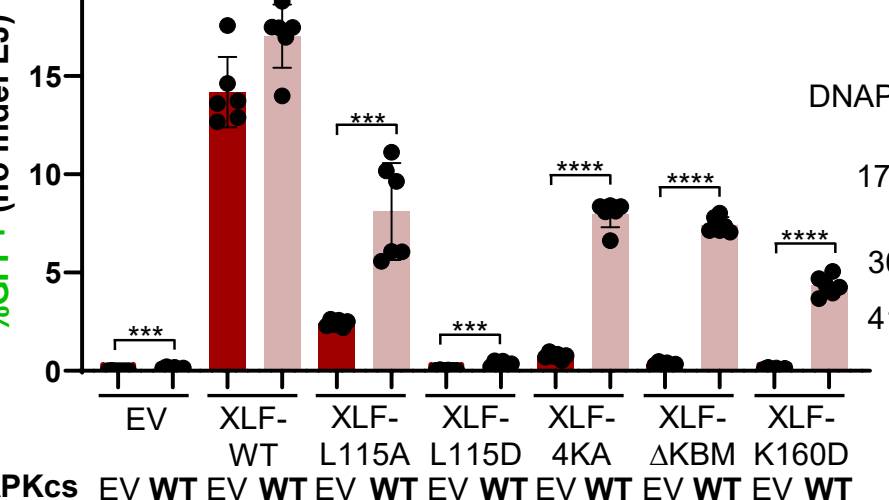

$X L F-K O / P R K D C-K O$

Cisneros-Aguirre et. al. Figure 5

DNAPKcs EV WT EV WT EV WT EV WT EV WT EV WT EV WT
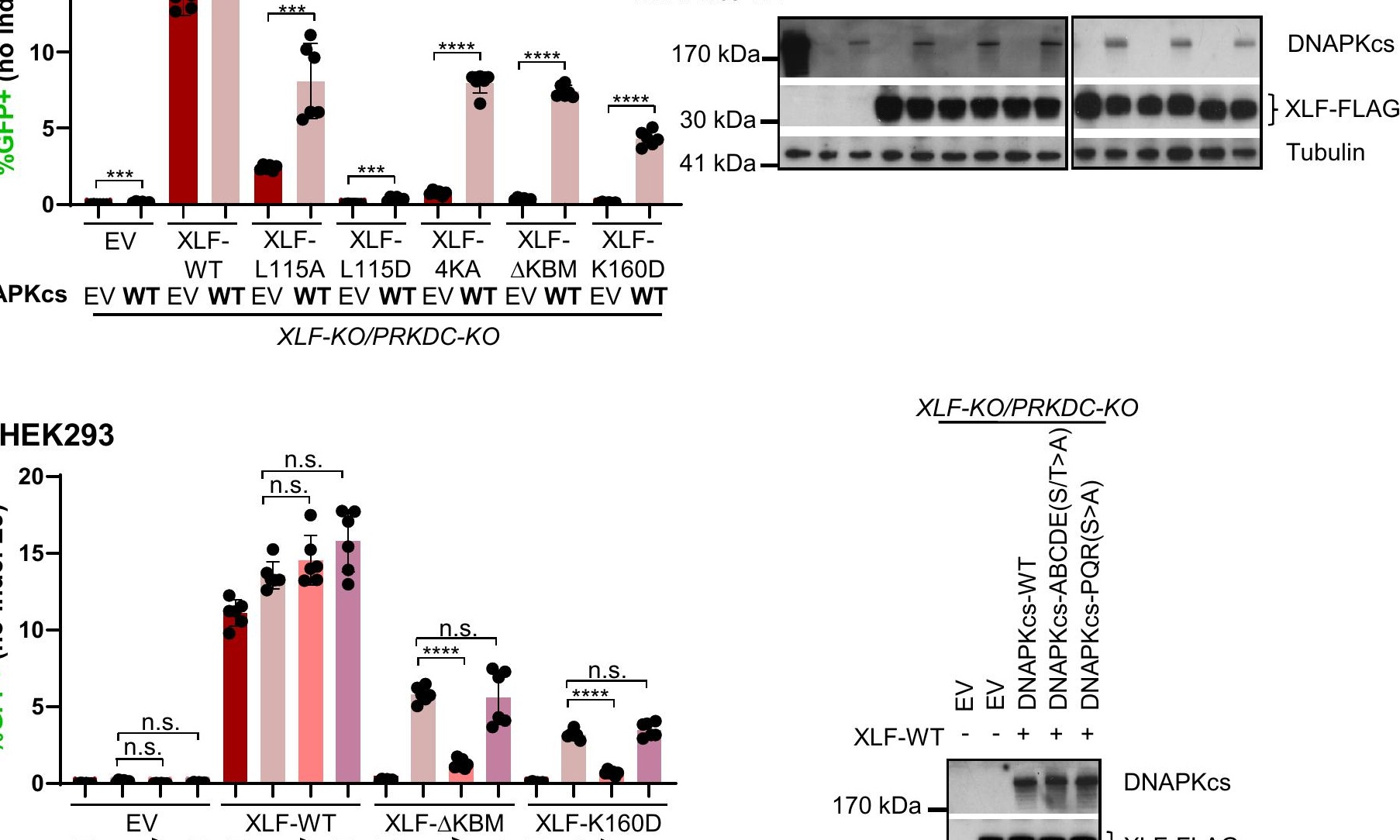

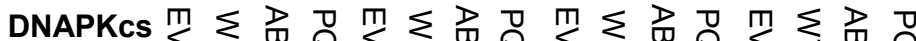

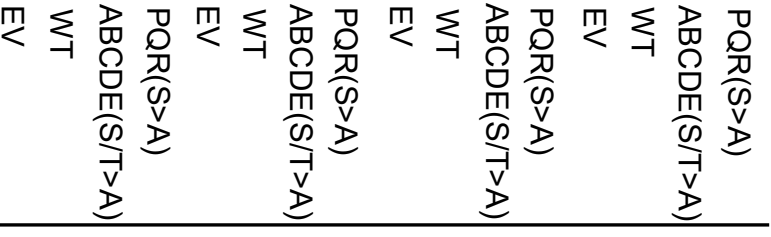

\section{$X L F-K O / P R K D C-K O$}


b $\quad$ Cisneros-Aguirre et. al. Figure 6
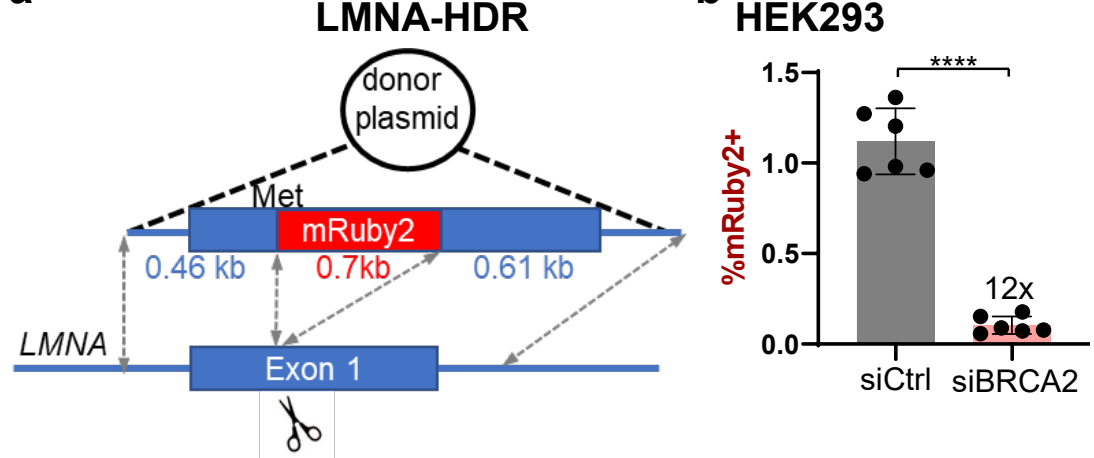
HEK293

C

HEK293

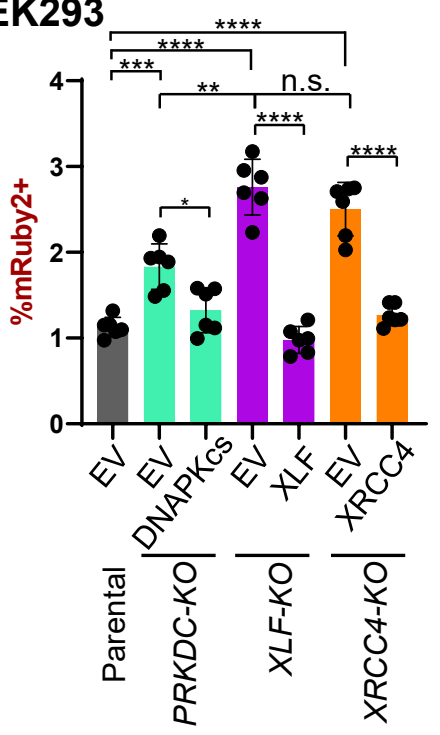

d HEK293
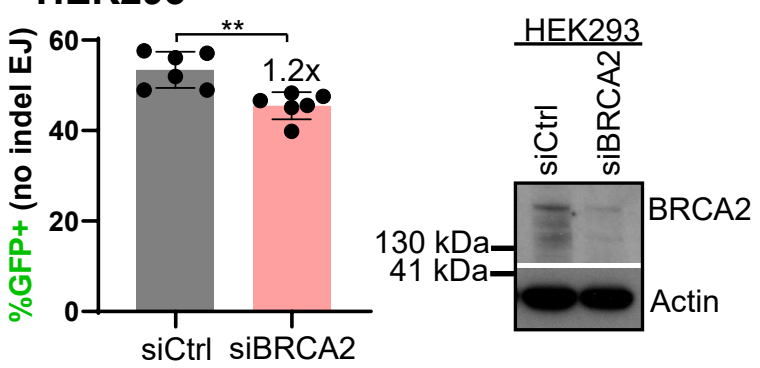

e

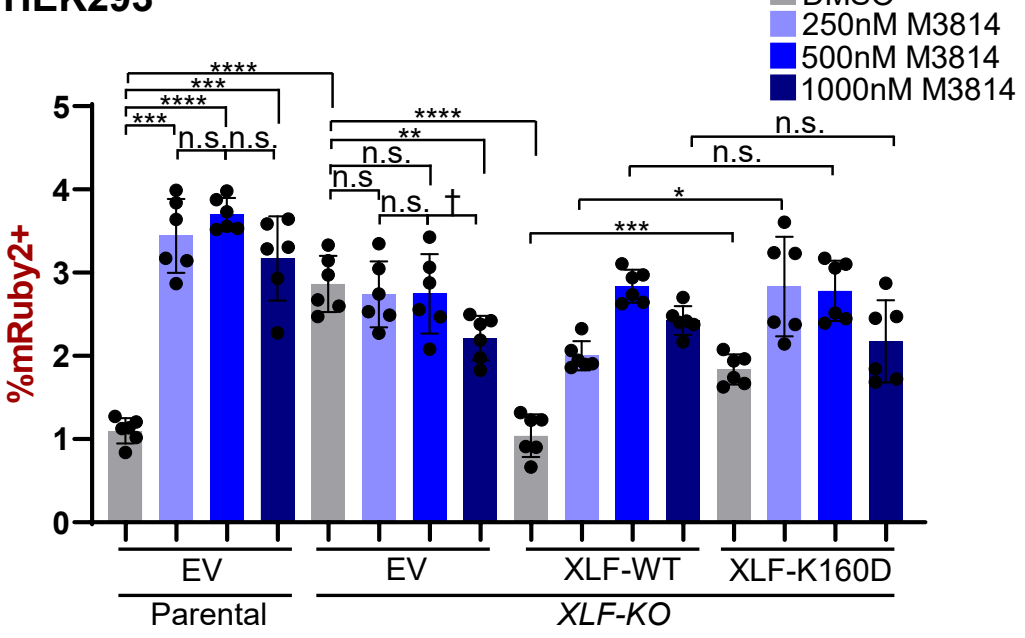

GFPd2-SV

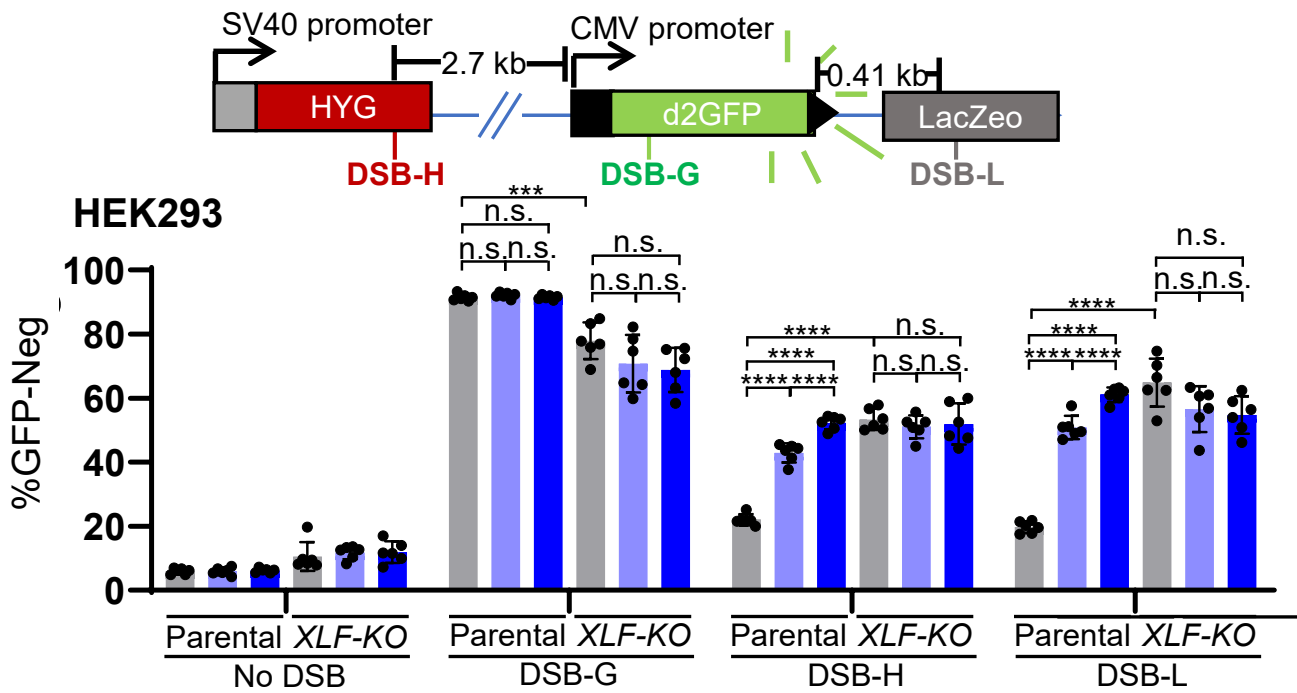

f HEK293 (stable)

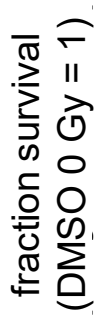

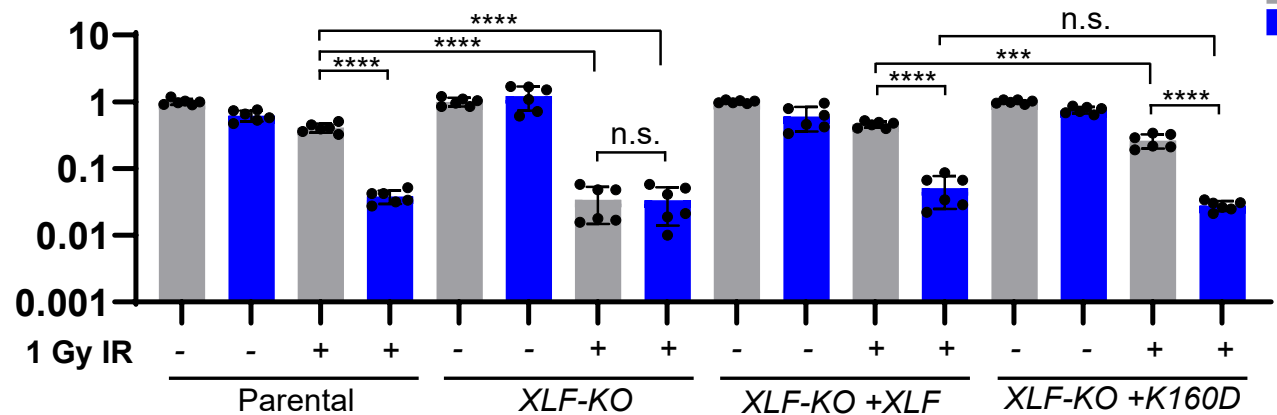

DMSO

250nM M3814

500nM M3814 
Long Range (LR)

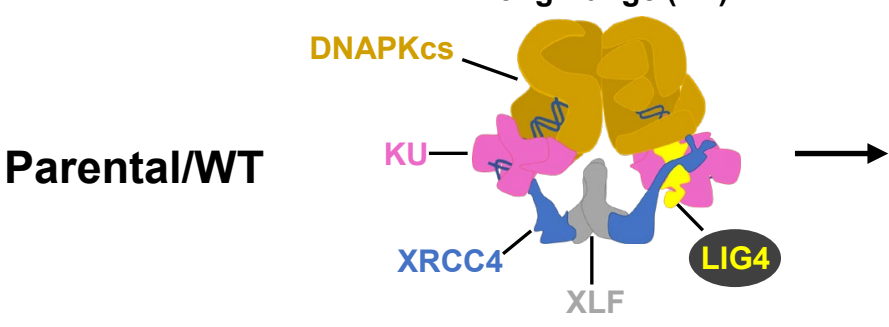

Blunt DSB EJ

PRKDC-KO

XLF-KO

w/ weakened XLF

(e.g., XLF-K160D)
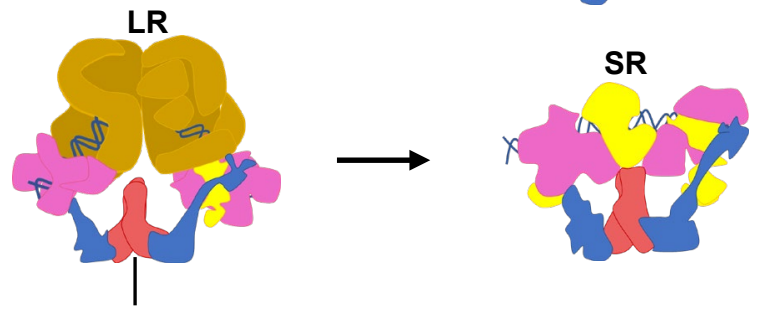

Weakened XLF

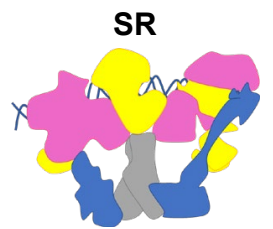
Short Range (SR)
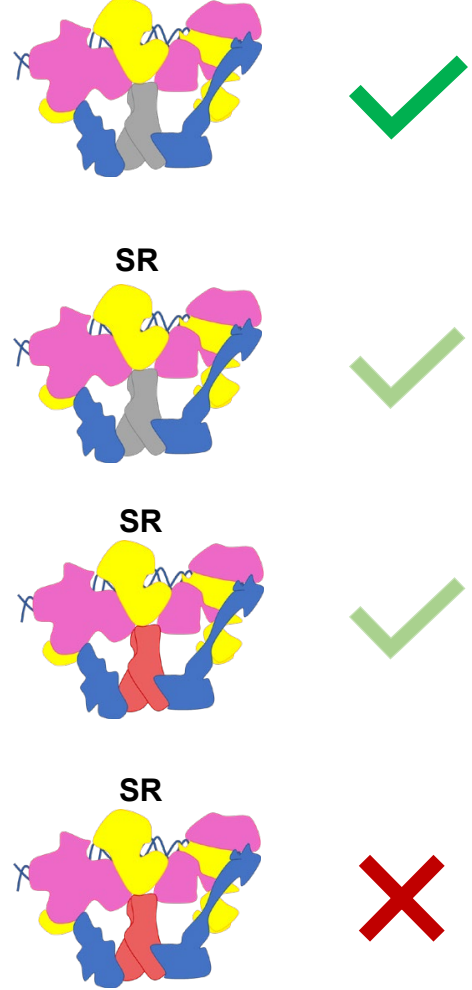

XLF-KO/PRKDC-KO

w/ weakened XLF

M3814 or

DNAPKcs-ABCDE(S/T>A)
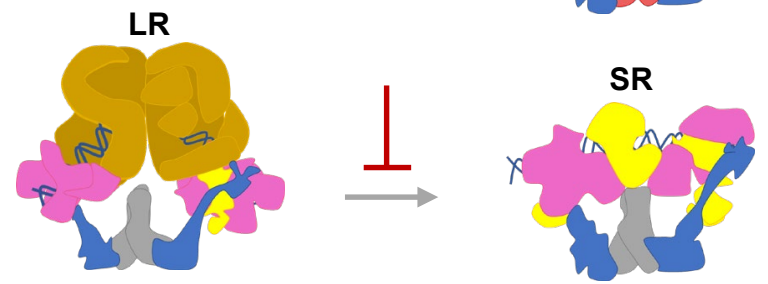

M3814 or

DNAPKcs-ABCDE(S/T>A) w/ weakened XLF
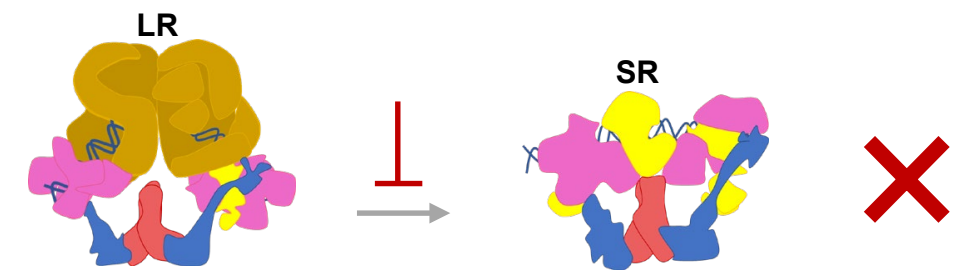